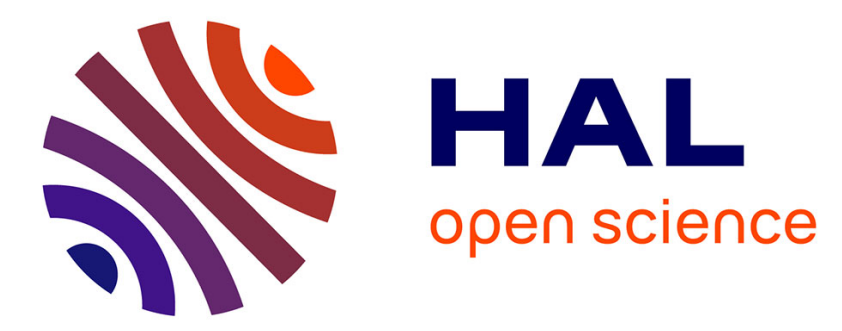

\title{
Spatial particle correlations in light nuclei. II Four-particle systems
}

\author{
P. Mei, P. van Isacker
}

\section{To cite this version:}

P. Mei, P. van Isacker. Spatial particle correlations in light nuclei. II Four-particle systems. Annals of Physics, 2012, 327, pp.1182-1201. 10.1016/j.aop.2011.12.003 . in2p3-00656026

\section{HAL Id: in2p3-00656026 \\ https://hal.in2p3.fr/in2p3-00656026}

Submitted on 3 Jan 2012

HAL is a multi-disciplinary open access archive for the deposit and dissemination of scientific research documents, whether they are published or not. The documents may come from teaching and research institutions in France or abroad, or from public or private research centers.
L'archive ouverte pluridisciplinaire HAL, est destinée au dépôt et à la diffusion de documents scientifiques de niveau recherche, publiés ou non, émanant des établissements d'enseignement et de recherche français ou étrangers, des laboratoires publics ou privés. 


\title{
Spatial particle correlations in light nuclei. II Four-particle systems
}

\author{
P. Mei and P. Van Isacker \\ GANIL, CEA/DSM-CNRS/IN2P3, BP 55027, F-14076 Caen Cedex 5, France
}

\begin{abstract}
The geometry of configurations of identical particles in a shell is extended from two to four particles. Results are derived for a general $\ell$ shell and particular attention is paid to the $p$ shell $(\ell=1)$, which is of relevance for the nucleus ${ }^{8}$ He. Expressions are given for the angular probability density in terms of the six angles between pairs of position vectors of the particles. The analysis of the $p$ shell reveals the existence of two classes of favored four-particle configurations, with three members each. The transition from one class to the other is governed by the nuclear dynamics and depends on the conflicting tendencies of the short-range nuclear interaction versus the spin-orbit splitting.
\end{abstract}

Key words: few-body systems, shell model, neutron distributions PACS: 21.45.+v, 21.60.Cs, 21.10.Gv

\section{Introduction}

In the first paper of this series [1], henceforth referred to as I, we argued that the di-neutron and cigar-like configurations, commonly conjectured to be observed in the ground state of ${ }^{6} \mathrm{He}$, can be understood as a consequence of the Pauli principle acting between two identical particles in the $p$ shell. Although the validity of this result is widely accepted by the nuclear physics community interested in halo phenomena [2], we pointed out in I that it could be understood from a simple perspective using an elementary version of the shell model. A particularly fruitful way to approach the problem proved to be through the use of an angular correlation function, involving the angle between the position vectors of the two particles, which lead an analytic insight into the geometric configurations of two particles in any shell, and in the $p$ shell in particular. 
In this paper we venture into lesser-known territory and, with the example of ${ }^{8} \mathrm{He}$ in mind, we tackle the problem of the geometry of four identical particles in a single- $\ell$ shell. Unlike the di-neutron and cigar-like configurations for ${ }^{6} \mathrm{He}$, which are well documented in the literature, to our knowledge comparatively few theoretical studies have appeared concerning the geometric structure of ${ }^{8}$ He. There exists a fairly old paper of Zhukov et al. [3] who briefly discuss the geometry of four identical particles in the $0 p_{3 / 2}$ shell. More recently, Hagino et al. [4] studied the structure of ${ }^{8} \mathrm{He}$ through a five-body calculation (the $\alpha$ particle and four neutrons) in a microscopic Hartree-Fock-Bogoliubov approach. In fact, these two studies yield a different geometry for ${ }^{8} \mathrm{He}$ : while Zhukov et al. [3] picture it as an $\alpha$ particle symmetrically surrounded by the four neutrons, Hagino et al. [4] find that these are grouped into two weakly interacting di-neutrons.

The purpose of the present paper is to extend the methods introduced in I, with the aim to obtain a geometric insight into the structure of four-particle systems. The approach starts from a basic version of the shell model in the framework of which it is possible to generalize the concept of the angular correlation function from two to four particles. Applied to ${ }^{8} \mathrm{He}$ (i.e., to four particles in the $p$ shell), this method leads in a natural way to the configurations that were encountered by Zhukov et al. [3] and by Hagino et al. [4] and, moreover, it establishes the conditions for one or the other configuration to be the dominant one in the ground state of ${ }^{8} \mathrm{He}$.

The outline of the paper is as follows. In Sect. 2 the method is explained for the particular and unique state with four identical particles in a $p_{3 / 2}$ shell. These results are then generalized in Sect. 3 to four identical particles in a single shell with angular momentum $\ell$ with particular emphasis on the case $\ell=1$ (the $p$ shell) which applies to ${ }^{8} \mathrm{He}$. In Sect. 4 the conclusions and perspectives of this work are formulated.

\section{The geometry of four identical particles in a $p_{3 / 2}$ shell}

We begin with a detailed study of the geometry of the case of four identical particles in the $0 p_{3 / 2}$ shell. In the limit of strong spin-orbit coupling and of large major-shell splittings (i.e., $0 s-0 p$ and $0 p-1 s 0 d$ ), this would be an adequate approximation for the ground state of ${ }^{8} \mathrm{He}$. The valence neutrons completely fill the $0 p_{3 / 2}$ shell and as a result there is only one possible state: $\left|0_{1}^{+}\right\rangle \equiv\left|\left(0 p_{3 / 2}\right)^{4} ; 0^{+}\right\rangle$. First, in Subsect. 2.1, the angular part of its probability density is derived and subsequently, in Subsect. 2.2, the spatial configurations that maximize this probability density are determined. The amount of detail provided in this section will pave the way for the treatment of more complicated configurations in subsequent sections. 


\subsection{Angular probability density of the $\left|\left(p_{3 / 2}\right)^{4} ; 0^{+}\right\rangle$state}

The Slater determinant for an isolated system of four identical particles in the $0 p_{3 / 2}$ shell is written as

$$
\frac{1}{\sqrt{4 !}}\left|\begin{array}{llll}
\psi_{3 / 2,3 / 2}(1) & \psi_{3 / 2,1 / 2}(1) & \psi_{3 / 2,-1 / 2}(1) & \psi_{3 / 2,-3 / 2}(1) \\
\psi_{3 / 2,3 / 2}(2) & \psi_{3 / 2,1 / 2}(2) & \psi_{3 / 2,-1 / 2}(2) & \psi_{3 / 2,-3 / 2}(2) \\
\psi_{3 / 2,3 / 2}(3) & \psi_{3 / 2,1 / 2}(3) & \psi_{3 / 2,-1 / 2}(3) & \psi_{3 / 2,-3 / 2}(3) \\
\psi_{3 / 2,3 / 2}(4) & \psi_{3 / 2,1 / 2}(4) & \psi_{3 / 2,-1 / 2}(4) & \psi_{3 / 2,-3 / 2}(4)
\end{array}\right|
$$

with $\psi_{3 / 2, m}(k)$ the single-particle wave function of particle $k$. The radial part is of the same form for all four single-particle wave functions and is equal to

$$
\sqrt{\frac{8}{3 \sqrt{\pi} b^{3}}}\left(\frac{r_{k}}{b}\right) \exp \left(-\frac{r_{k}^{2}}{2 b^{2}}\right)
$$

with $b$ the length of the harmonic oscillator, $b=\sqrt{\hbar / m \omega}$, where $m$ is the mass of the particles and $\omega$ the frequency of the oscillator. The angular and spin parts of the single-particle wave functions are

$$
\begin{aligned}
\psi_{3 / 2,3 / 2}(k) & =Y_{11}\left(\Omega_{k}\right) \chi_{+1 / 2}(k), \\
\psi_{3 / 2,1 / 2}(k) & =\sqrt{\frac{1}{3}} Y_{11}\left(\Omega_{k}\right) \chi_{-1 / 2}(k)+\sqrt{\frac{2}{3}} Y_{10}\left(\Omega_{k}\right) \chi_{+1 / 2}(k), \\
\psi_{3 / 2,-1 / 2}(k) & =\sqrt{\frac{2}{3}} Y_{10}\left(\Omega_{k}\right) \chi_{-1 / 2}(k)+\sqrt{\frac{1}{3}} Y_{1-1}\left(\Omega_{k}\right) \chi_{+1 / 2}(k), \\
\psi_{3 / 2,-3 / 2}(k) & =Y_{1-1}\left(\Omega_{k}\right) \chi_{-1 / 2}(k),
\end{aligned}
$$

where $Y_{\ell m}\left(\Omega_{k}\right)$ are spherical harmonics in terms of the polar angle $\theta_{k}$ and the azimuthal angle $\phi_{k}$ (collectively denoted as $\Omega_{k}$ ) of particle $k$. For the $p$ shell the spherical harmonics assume the explicit expressions

$$
\begin{aligned}
Y_{10}(\Omega) & =\sqrt{\frac{3}{4 \pi}} \cos \theta=\sqrt{\frac{3}{4 \pi}} \frac{z}{r}, \\
Y_{1 \pm 1}(\Omega) & =\mp \sqrt{\frac{3}{8 \pi}} \sin \theta e^{ \pm i \phi}=\mp \sqrt{\frac{3}{8 \pi}} \frac{x \pm i y}{r} .
\end{aligned}
$$

Furthermore, $\chi_{ \pm 1 / 2}$ are spinors for a spin-1/2 particle,

$$
\chi_{+1 / 2}=\left(\begin{array}{l}
1 \\
0
\end{array}\right), \quad \chi_{-1 / 2}=\left(\begin{array}{l}
0 \\
1
\end{array}\right)
$$


As only the angular part of the probability density is of concern here, the radial part (2) is suppressed in the following and the subsequent discussion is therefore valid for any $p$ shell. ${ }^{1}$ The angular part of the wave function is obtained by inserting the explicit expressions (3) into the Slater determinant (1), leading to a sum of products of a spatial wave function for the four particles, $\Phi_{m_{1} m_{2} m_{3} m_{4}}\left(\bar{r}_{1}, \bar{r}_{2}, \bar{r}_{3}, \bar{r}_{4}\right)$, with a spin part $\chi_{m_{1}}(1) \chi_{m_{2}}(2) \chi_{m_{3}}(3) \chi_{m_{4}}(4)$. There are $2^{4}-2=14$ such products since four particles in a $p$ shell cannot all have the same spin projection, which excludes the products $\chi_{+1 / 2}(1) \chi_{+1 / 2}(2) \chi_{+1 / 2}(3) \chi_{+1 / 2}(4)$ and $\chi_{-1 / 2}(1) \chi_{-1 / 2}(2) \chi_{-1 / 2}(3) \chi_{-1 / 2}(4)$. The sum can be written as

$$
\begin{aligned}
& \Psi_{0_{1}^{+}}(1,2,3,4) \\
& =\frac{f}{r_{1} r_{2} r_{3} r_{4}} \sum_{m_{i}} \Phi_{m_{1} m_{2} m_{3} m_{4}}\left(\bar{r}_{1}, \bar{r}_{2}, \bar{r}_{3}, \bar{r}_{4}\right) \chi_{m_{1}}(1) \chi_{m_{2}}(2) \chi_{m_{3}}(3) \chi_{m_{4}}(4),
\end{aligned}
$$

with

$$
f=\left(\frac{1}{2} \sqrt{\frac{3}{2 \pi}}\right)^{4} \frac{1}{\sqrt{4 !}} \frac{4}{3} .
$$

In Appendix A it is shown how the expressions (4) of the spherical harmonics in cartesian coordinates lead to the following angular part of the spatial probability density associated with the wave function (6):

$$
\begin{aligned}
& \mathcal{P}_{0_{1}^{+}}\left(\Omega_{1}, \Omega_{2}, \Omega_{3}, \Omega_{4}\right) \\
& \begin{array}{r}
=\frac{3}{1024 \pi^{4}} \frac{1}{r_{1}^{2} r_{2}^{2} r_{3}^{2} r_{4}^{2}}\left(\left(\bar{r}_{12} \cdot \bar{r}_{34}\right)^{2}+\left(\bar{r}_{13} \cdot \bar{r}_{24}\right)^{2}+\left(\bar{r}_{14} \cdot \bar{r}_{23}\right)^{2}+\right. \\
\left.\quad+\left|\bar{r}_{12} \times \bar{r}_{34}\right|^{2}+\left|\bar{r}_{13} \times \bar{r}_{24}\right|^{2}+\left|\bar{r}_{14} \times \bar{r}_{23}\right|^{2}\right),
\end{array}
\end{aligned}
$$

where the notation

$$
\bar{r}_{i j}=\left(x_{i j}, y_{i j}, z_{i j}\right)=\bar{r}_{i} \times \bar{r}_{j}=\left(y_{i} z_{j}-y_{j} z_{i}, z_{i} x_{j}-z_{j} x_{i}, x_{i} y_{j}-y_{i} x_{j}\right),
$$

is used. Let $\theta_{i j}$ denote the angle between $\bar{r}_{i}$ and $\bar{r}_{j}$, and $\theta_{i j, k l}$ the angle between $\bar{r}_{i j}$ and $\bar{r}_{k l}$. The dot product $\bar{r}_{i j} \cdot \bar{r}_{k l}$ and the length of the cross product $\bar{r}_{i j} \times$ $\bar{r}_{k l}$ are equal to $r_{i} r_{j} r_{k} r_{l} \sin \theta_{i j} \sin \theta_{k l} \cos \theta_{i j, k l}$ and $r_{i} r_{j} r_{k} r_{l} \sin \theta_{i j} \sin \theta_{k l} \sin \theta_{i j, k l}$, respectively. As a result, the identity

$$
\left(\bar{r}_{i j} \cdot \bar{r}_{k l}\right)^{2}+\left|\bar{r}_{i j} \times \bar{r}_{k l}\right|^{2}=\left(r_{i} r_{j} r_{k} r_{l} \sin \theta_{i j} \sin \theta_{k l}\right)^{2},
$$

can be used to derive the final result ${ }^{2}$

1 For this reason the radial quantum number shall be omitted henceforth, except when specific reference is made to ${ }^{6} \mathrm{He}$ or ${ }^{8} \mathrm{He}$.

2 The abbreviations $\sin ^{2} \theta$ and $\cos ^{2} \theta$ are used to denote $(\sin \theta)^{2}$ and $(\cos \theta)^{2}$. 


$$
\begin{aligned}
& \mathcal{P}_{0_{1}^{+}}\left(\theta_{12}, \theta_{13}, \theta_{14}, \theta_{23}, \theta_{24}, \theta_{34}\right) \\
& =c\left(\sin ^{2} \theta_{13} \sin ^{2} \theta_{24}+\sin ^{2} \theta_{14} \sin ^{2} \theta_{23}+\sin ^{2} \theta_{12} \sin ^{2} \theta_{34}\right),
\end{aligned}
$$

where the constant $c=3 \cdot 2^{-10} \pi^{-4}$ is introduced for subsequent convenience.

We conclude that the angular part of the probability density of the state with four identical particles in a $p_{3 / 2}$ shell has the simple geometrical structure given by Eq. (11), a result which coincides with that of Zhukov et al. [3]. In the case of ${ }^{8} \mathrm{He}$, it is a function of the six relative angles formed by the four vectors connecting the four neutrons and the $\alpha$-particle core, and it is invariant under permutation of the valence neutrons. Furthermore, it is independent of the angles $\theta_{i j, k l}$ between the planes formed by pairs of neutrons and the $\alpha$-particle core. It might seem that the geometric configurations that maximize the probability density of the state $\left|0_{1}^{+}\right\rangle$are trivially obtained from Eq. (11). This is not the case, however, because only five of the six angles $\theta_{i j}$ are independent and the problem therefore needs a closer analysis which is presented in the next subsection.

\subsection{Favored geometric configurations of the $\left|\left(p_{3 / 2}\right)^{4} ; 0^{+}\right\rangle$state}

The purpose of this subsection is to determine the geometric configurations for which the angular probability density of the $\left|0_{1}^{+}\right\rangle$state is maximal. As mentioned at the end of the previous subsection, the six relative angles $\theta_{i j}$ are not independent and one of the angles can be deduced from the others. In addition, there are constraints on the five chosen independent angles. As a result, it is more appropriate to express the probability density (11) in terms of the azimuthal and polar angles of the particles' spherical coordinates. There are eight such angles, only five of which are independent because of rotational invariance. One can fix the position of one of the particles, say particle 4 , at $\theta_{4}=0$ and $\phi_{4}=0$, and require that another particle, say particle 1 , lies in the $\phi_{1}=0$ plane, so that five independent variables remain: $\theta_{1}, \theta_{2}, \theta_{3}, \phi_{2}$ and $\phi_{3}$. For clarity, $\phi_{1}$ is kept for the moment and Eq. (11) is rewritten as

$$
\begin{aligned}
& \mathcal{P}_{0_{1}^{+}}\left(\theta_{1}, \theta_{2}, \theta_{3}, \phi_{1}, \phi_{2}, \phi_{3}\right) \\
& =c\left\{\sin ^{2} \theta_{1}\left[1-\left(\cos \theta_{2} \cos \theta_{3}+\cos \left(\phi_{2}-\phi_{3}\right) \sin \theta_{2} \sin \theta_{3}\right)^{2}\right]\right. \\
& \quad+\sin ^{2} \theta_{2}\left[1-\left(\cos \theta_{1} \cos \theta_{3}+\cos \left(\phi_{1}-\phi_{3}\right) \sin \theta_{1} \sin \theta_{3}\right)^{2}\right] \\
& \left.\quad+\sin ^{2} \theta_{3}\left[1-\left(\cos \theta_{1} \cos \theta_{2}+\cos \left(\phi_{1}-\phi_{2}\right) \sin \theta_{1} \sin \theta_{2}\right)^{2}\right]\right\},
\end{aligned}
$$


where use is made of the property

$$
\cos \theta_{i j}=\cos \theta_{i} \cos \theta_{j}+\cos \left(\phi_{i}-\phi_{j}\right) \sin \theta_{i} \sin \theta_{j}
$$

which implies $\sin ^{2} \theta_{i 4}=\sin ^{2} \theta_{i}$. The absolute maximum of this function occurs at a critical point where the first partial derivatives with respect to the five variables $\theta_{1}, \theta_{2}, \theta_{3}, \phi_{2}$ and $\phi_{3}$ vanish. However, the search for the absolute maximum will be complicated by the fact that function (12) abounds in local extrema and saddle points where the first partial derivatives also vanish.

The condition $\partial_{\theta_{1}} \mathcal{P}_{0_{1}^{+}}=0$ leads to

$$
\begin{aligned}
& \cos ^{2} \theta_{23} \sin ^{2} \theta_{1}+\cos ^{2} \theta_{13} \sin ^{2} \theta_{2}+\cos ^{2} \theta_{12} \sin ^{2} \theta_{3} \\
& -\cos \theta_{13} \frac{\cos \theta_{3} \sin ^{2} \theta_{2}}{\cos \theta_{1}}-\cos \theta_{12} \frac{\cos \theta_{2} \sin ^{2} \theta_{3}}{\cos \theta_{1}}-\sin ^{2} \theta_{1}=0 .
\end{aligned}
$$

Similarly, the conditions $\partial_{\theta_{2}} \mathcal{P}_{0_{1}^{+}}=0$ and $\partial_{\theta_{3}} \mathcal{P}_{0_{1}^{+}}=0$ lead to

$$
\begin{aligned}
& \cos ^{2} \theta_{23} \sin ^{2} \theta_{1}+\cos ^{2} \theta_{13} \sin ^{2} \theta_{2}+\cos ^{2} \theta_{12} \sin ^{2} \theta_{3} \\
& -\cos \theta_{23} \frac{\cos \theta_{3} \sin ^{2} \theta_{1}}{\cos \theta_{2}}-\cos \theta_{12} \frac{\cos \theta_{1} \sin ^{2} \theta_{3}}{\cos \theta_{2}}-\sin ^{2} \theta_{2}=0,
\end{aligned}
$$

and

$$
\begin{aligned}
& \cos ^{2} \theta_{23} \sin ^{2} \theta_{1}+\cos ^{2} \theta_{13} \sin ^{2} \theta_{2}+\cos ^{2} \theta_{12} \sin ^{2} \theta_{3} \\
& -\cos \theta_{13} \frac{\cos \theta_{1} \sin ^{2} \theta_{2}}{\cos \theta_{3}}-\cos \theta_{23} \frac{\cos \theta_{2} \sin ^{2} \theta_{1}}{\cos \theta_{3}}-\sin ^{2} \theta_{3}=0 .
\end{aligned}
$$

The latter two equations can also be obtained by permuting particles 1 and 2, and particles 1 and 3 in Eq. (14), respectively, which is obvious given that function (12) is invariant under permutation of any two particles. The condition $\partial_{\phi_{1}} \mathcal{P}_{0_{1}^{+}}=0$ leads to

$$
\sin \theta_{1} \sin \theta_{2} \sin \theta_{3}\left[\sin \left(\phi_{1}-\phi_{3}\right) \sin \theta_{2} \cos \theta_{13}+\sin \left(\phi_{1}-\phi_{2}\right) \sin \theta_{3} \cos \theta_{12}\right]=0,
$$

and, by permutation, the equations for the other two derivatives are

$$
\sin \theta_{1} \sin \theta_{2} \sin \theta_{3}\left[\sin \left(\phi_{2}-\phi_{3}\right) \sin \theta_{1} \cos \theta_{23}-\sin \left(\phi_{1}-\phi_{2}\right) \sin \theta_{3} \cos \theta_{12}\right]=0,
$$

and

$$
\sin \theta_{1} \sin \theta_{2} \sin \theta_{3}\left[\sin \left(\phi_{1}-\phi_{3}\right) \sin \theta_{2} \cos \theta_{13}+\sin \left(\phi_{2}-\phi_{3}\right) \sin \theta_{1} \cos \theta_{23}\right]=0
$$

Equations (17), (18) and (19) are not independent. One obvious possible solution to these equations is $\sin \theta_{1}=0$ or $\sin \theta_{2}=0$ or $\sin \theta_{3}=0$. In each of those 
cases the maximum of (12) is $2 c$ and corresponds to one of the solutions that will be found later. Other possible solutions, with $\sin \theta_{1} \neq 0$ and $\sin \theta_{2} \neq 0$ and $\sin \theta_{3} \neq 0$, must be due to the vanishing of all three terms between square brackets in Eqs. (17), (18) and (19). An obvious solution of that system of three equations satisfies

$$
\sin \theta_{1} \cos \theta_{23}=\sin \theta_{2} \cos \theta_{13}=-\sin \theta_{3} \cos \theta_{12}
$$

and

$$
\sin \left(\phi_{1}-\phi_{2}\right)=\sin \left(\phi_{1}-\phi_{3}\right)=-\sin \left(\phi_{2}-\phi_{3}\right)
$$

Signs can be chosen differently $\left[e . g ., \sin \left(\phi_{1}-\phi_{2}\right)=\sin \left(\phi_{1}-\phi_{3}\right)=\sin \left(\phi_{2}-\right.\right.$ $\left.\phi_{3}\right)$ ] but this leads to essentially the same results as obtained below. Since the probability density (11) is invariant under any permutation of the four particles, one may choose, without loss of generality, $0 \leq \phi_{1} \leq \phi_{2} \leq \phi_{3}<2 \pi$. Furthermore, for $\phi_{1}=0$, Eq. (21) reduces to

$$
\sin \phi_{2}=\sin \phi_{3}=\sin \left(\phi_{2}-\phi_{3}\right)
$$

Subject to the condition $0 \leq \phi_{2} \leq \phi_{3}<2 \pi$, these equations admit the solutions

$$
\left(\phi_{2}, \phi_{3}\right)=(0,0), \quad\left(\phi_{2}, \phi_{3}\right)=(0, \pi), \quad\left(\phi_{2}, \phi_{3}\right)=(\pi, \pi)
$$

as well as

$$
\left(\phi_{2}, \phi_{3}\right)=\left(\frac{4 \pi}{3}, \frac{5 \pi}{3}\right)
$$

For reasons that will become apparent in the following, solutions (23) and (24) will be referred to as "great-circle" and "tetrahedral" configurations, respectively. These will be discussed separately in the subsequent subsections.

\subsubsection{Great-circle configurations}

If $\phi_{1}=\phi_{2}=\phi_{3}=\phi_{4}=0$ [the first solution in Eq. (23)], the four particles are in a plane that passes through the center, that is, they are on a great circle. As a result, expression (12) can be simplified to give

$$
\begin{aligned}
& \mathcal{P}_{0_{1}^{+}}\left(\theta_{1}, \theta_{2}, \theta_{3}, 0,0,0\right) \\
& =c\left[\sin ^{2} \theta_{1} \sin ^{2}\left(\theta_{2}-\theta_{3}\right)+\sin ^{2} \theta_{2} \sin ^{2}\left(\theta_{1}-\theta_{3}\right)+\sin ^{2} \theta_{3} \sin ^{2}\left(\theta_{1}-\theta_{2}\right)\right] .
\end{aligned}
$$

Partial derivation with respect to $\theta_{i}$ leads to the following set of equations:

$$
\begin{aligned}
& {\left[\sin \theta_{1} \sin \left(\theta_{2}-\theta_{3}\right)+\sin \theta_{2} \sin \left(\theta_{1}-\theta_{3}\right)\right] \sin \left(\theta_{1}+\theta_{2}-\theta_{3}\right)=0,} \\
& {\left[\sin \theta_{1} \sin \left(\theta_{2}-\theta_{3}\right)-\sin \theta_{3} \sin \left(\theta_{1}-\theta_{2}\right)\right] \sin \left(\theta_{2}-\theta_{3}-\theta_{1}\right)=0,} \\
& {\left[\sin \theta_{2} \sin \left(\theta_{1}-\theta_{3}\right)+\sin \theta_{3} \sin \left(\theta_{1}-\theta_{2}\right)\right] \sin \left(\theta_{1}-\theta_{2}-\theta_{3}\right)=0}
\end{aligned}
$$




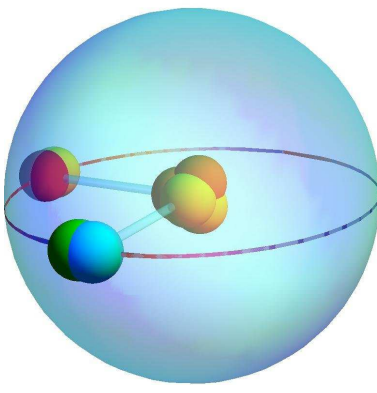

(a)

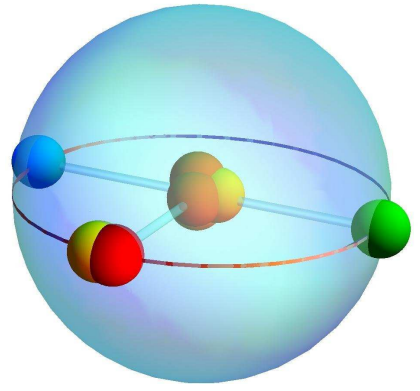

(b)

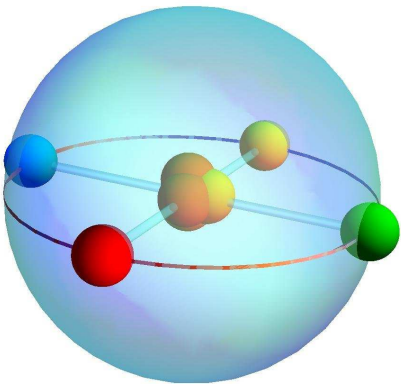

(c)

Fig. 1. The great-circle configurations that correspond to a local maximum of the probability density of the $\left|\left(p_{3 / 2}\right)^{4} ; 0^{+}\right\rangle$state. The configurations shown correspond to (a) $\left(\theta_{1}, \theta_{2}, \theta_{3}\right)=\left(\frac{1}{2} \pi, \frac{1}{2} \pi, 0\right)$ and $\left(\phi_{1}, \phi_{2}, \phi_{3}\right)=(0,0,0)$, (b) $\left(\theta_{1}, \theta_{2}, \theta_{3}\right)=\left(\pi, \frac{1}{2} \pi, \frac{1}{2} \pi\right)$ and $\left(\phi_{1}, \phi_{2}, \phi_{3}\right)=(0,0,0)$, and (c) $\left(\theta_{1}, \theta_{2}, \theta_{3}\right)=\left(\pi, \frac{1}{2} \pi, \frac{1}{2} \pi\right)$ and $\left(\phi_{1}, \phi_{2}, \phi_{3}\right)=(0,0, \pi)$, always with $\theta_{4}=\phi_{4}=0$.

Since all $\phi_{i}$ are equal and the probability density (11) is invariant under permutation, one may again require, without loss of generality, $0 \leq \theta_{3} \leq \theta_{2} \leq \theta_{1} \leq \pi$. A number of trivial solutions of Eq. (26) occur, namely, (1) $\theta_{1}=\theta_{2}=\theta_{3}$ or (2) any two of the angles $\theta_{i}$ are equal to 0 or $\pi$. These solutions are not of interest since they correspond to a vanishing probability density which clearly is ruled out as a maximum. The two remaining solutions satisfy

$$
\left(\theta_{1}, \theta_{2}, \theta_{3}\right)=\left(\frac{\pi}{2}, \frac{\pi}{2}, 0\right), \quad\left(\theta_{1}, \theta_{2}, \theta_{3}\right)=\left(\pi, \frac{\pi}{2}, \frac{\pi}{2}\right)
$$

and therefore lead to the two configurations

$$
\begin{array}{ll}
\text { (a) }:\left(\theta_{1}, \theta_{2}, \theta_{3}\right) & =\left(\frac{\pi}{2}, \frac{\pi}{2}, 0\right), \quad\left(\phi_{1}, \phi_{2}, \phi_{3}\right)=(0,0,0), \\
\text { (b) }:\left(\theta_{1}, \theta_{2}, \theta_{3}\right) & =\left(\pi, \frac{\pi}{2}, \frac{\pi}{2}\right), \quad\left(\phi_{1}, \phi_{2}, \phi_{3}\right)=(0,0,0),
\end{array}
$$

always with $\theta_{4}=\phi_{4}=0$. The probability density (12) reaches an extremum value $2 c$ at these two configurations which are represented in Figs. 1(a) and 1(b). Since function (12) is inversion-invariant with respect to the origin, the coordinates $(\theta, \phi)$ of any particle can be changed to $(\pi-\theta, \pi+\phi)$ without altering its value. Thus, by inverting particle positions in the configurations shown in Fig. 1(a) or 1(b), a third extremal configuration is found to be

$$
\text { (c) : }\left(\theta_{1}, \theta_{2}, \theta_{3}\right)=\left(\pi, \frac{\pi}{2}, \frac{\pi}{2}\right), \quad\left(\phi_{1}, \phi_{2}, \phi_{3}\right)=(0,0, \pi)
$$

as shown in Fig. 1(c).

The maximum configurations for the second and the third solution in Eq. (23), $\left(\phi_{1}, \phi_{2}, \phi_{3}\right)=(0,0, \pi)$ and $(0, \pi, \pi)$, can be obtained in the same manner and 
turn out to be equivalent to those that have just been found.

One still needs to verify the character of the different extrema, that is, to check whether they are maxima, minima or saddle points. To do so, it is necessary to calculate the Hessian matrix

$$
\left(\begin{array}{cccccc}
\partial_{\theta_{1}^{2}}^{2} \mathcal{P}_{0_{1}^{+}} & \partial_{\theta_{1} \theta_{2}}^{2} \mathcal{P}_{0_{1}^{+}} & \partial_{\theta_{1} \theta_{3}}^{2} \mathcal{P}_{0_{1}^{+}} & \partial_{\theta_{1} \phi_{2}}^{2} \mathcal{P}_{0_{1}^{+}} & \partial_{\theta_{1} \phi_{3}}^{2} \mathcal{P}_{0_{1}^{+}} \\
\partial_{\theta_{2} \theta_{1}}^{2} \mathcal{P}_{0_{1}^{+}} & \partial_{\theta_{2}^{2}}^{2} \mathcal{P}_{0_{1}^{+}} & \partial_{\theta_{2} \theta_{3}}^{2} \mathcal{P}_{0_{1}^{+}} & \partial_{\theta_{2} \phi_{2}}^{2} \mathcal{P}_{0_{1}^{+}} & \partial_{\theta_{2} \phi_{3}}^{2} \mathcal{P}_{0_{1}^{+}} \\
\partial_{\theta_{3} \theta_{1}}^{2} \mathcal{P}_{0_{1}^{+}} & \partial_{\theta_{3} \theta_{2}}^{2} \mathcal{P}_{0_{1}^{+}} & \partial_{\theta_{3}^{2}}^{2} \mathcal{P}_{0_{1}^{+}} & \partial_{\theta_{3} \phi_{2}}^{2} \mathcal{P}_{0_{1}^{+}} & \partial_{\theta_{3} \phi_{3}}^{2} \mathcal{P}_{0_{1}^{+}} \\
\partial_{\phi_{2} \theta_{1}}^{2} \mathcal{P}_{0_{1}^{+}} & \partial_{\phi_{2} \theta_{2}}^{2} \mathcal{P}_{0_{1}^{+}} & \partial_{\phi_{2} \theta_{3}}^{2} \mathcal{P}_{0_{1}^{+}} & \partial_{\phi_{2}^{2}}^{2} \mathcal{P}_{0_{1}^{+}} & \partial_{\phi_{2} \phi_{3}}^{2} \mathcal{P}_{0_{1}^{+}} \\
\partial_{\phi_{3} \theta_{1}}^{2} \mathcal{P}_{0_{1}^{+}} & \partial_{\phi_{3} \theta_{2}}^{2} \mathcal{P}_{0_{1}^{+}} & \partial_{\phi_{3} \theta_{3}}^{2} \mathcal{P}_{0_{1}^{+}} & \partial_{\phi_{3} \phi_{2}}^{2} \mathcal{P}_{0_{1}^{+}} & \partial_{\phi_{3}^{2}}^{2} \mathcal{P}_{0_{1}^{+}}
\end{array}\right)
$$

for each of the configurations in Fig. 1, which leads to the matrices

(a)

$$
\left(\begin{array}{rrrrr}
-4 & 0 & 2 & 0 & 0 \\
0 & -4 & 2 & 0 & 0 \\
2 & 2 & -4 & 0 & 0 \\
0 & 0 & 0 & 0 & 0 \\
0 & 0 & 0 & 0 & 0
\end{array}\right),\left(\begin{array}{rrrrr}
-4 & -2 & 2 & 0 & 0 \\
-2 & -4 & 0 & 0 & 0 \\
2 & 0 & -4 & 0 & 0 \\
0 & 0 & 0 & 0 & 0 \\
0 & 0 & 0 & 0 & 0
\end{array}\right),\left(\begin{array}{rrrrr}
-4-2 & 2 & 0 & 0 \\
-2 & -4 & 0 & 0 & 0 \\
2 & 0 & -4 & 0 & 0 \\
0 & 0 & 0 & 0 & 0 \\
0 & 0 & 0 & 0 & 0
\end{array}\right) .
$$

A sufficient condition for a function to have a local maximum at a point where all its first derivatives are zero is that the Hessian matrix is negative definite at that point. However, the matrices for the great-circle configurations are all negative semi-definite, which is inconclusive. Indeed, if one of the $\theta_{i}$, say $\theta_{1}$, equals 0 or $\pi$, function (12) reduces to $2 c \sin ^{2} \theta_{2} \sin ^{2} \theta_{3}$ and is independent of $\phi_{2}$ and $\phi_{3}$. As a consequence, if the polar angles $\theta_{i}$ are chosen as they are in the three great-circle configurations found above, the probability density is independent of azimuthal angles, that is, the four particles can rotate around the $z$ axis and retain the same probability density value $2 c$. As an illustration of this particularity, some configurations with the same polar angles as in the great-circle configurations but randomly chosen $\phi_{i}$ are shown in Fig. 2.

On the other hand, the sub-matrices comprised only of second derivatives with respect to $\theta_{1}, \theta_{2}$ and $\theta_{3}$ in the matrices (31) are negative definite. We conclude that the great-circle configurations in Fig. 1 are local, improper maxima of Eq. (12). Such cannot be said of configurations in Fig. 2 with randomly chosen $\phi_{i}$ because the submatrix of second partial derivatives with respect to the $\theta_{i}$ may not be negative definite. 


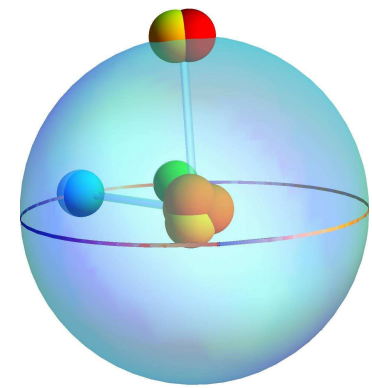

(a)

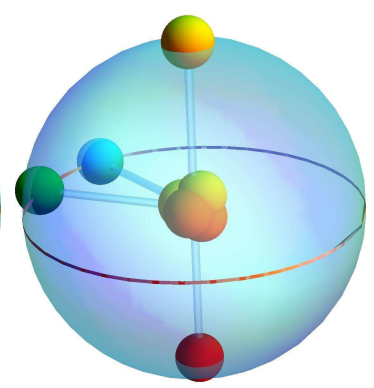

(b)

Fig. 2. Configurations with $\theta_{1}=0$ or $\pi$ but with random $\phi_{2}, \phi_{3}$. The two different configurations shown correspond to (a) $\left(\theta_{1}, \theta_{2}, \theta_{3}\right)=\left(0, \frac{1}{2} \pi, \frac{1}{2} \pi\right)$ and (b) $\left(\theta_{1}, \theta_{2}, \theta_{3}\right)=\left(\pi, \frac{1}{2} \pi, \frac{1}{2} \pi\right)$.

\subsubsection{Tetrahedral configurations}

Tetrahedral configurations are obtained if the second type of solution with $\left(\phi_{1}, \phi_{2}, \phi_{3}\right)=\left(0, \frac{4}{3} \pi, \frac{5}{3} \pi\right)$, as obtained in Eq. (24), is adopted. Conditions on the polar angles $\theta_{i}$ can be obtained by inserting the explicit values of the azimuthal angles $\phi_{i}$ into Eqs. (20) after use of the property (13), leading to

$$
\cos \theta_{3} \sin \left(\theta_{1}-\theta_{2}\right)=\cos \theta_{2} \sin \left(\theta_{1}+\theta_{3}\right)=\cos \theta_{1} \sin \left(\theta_{2}+\theta_{3}\right)=0
$$

These equations admit three classes of solutions. In the first, all angles $\theta_{i}$ are either 0 or $\pi$; these points can be discarded as a maxima since the probability density (12) vanishes in them. In the second class, all angles $\theta_{i}(i=1,2,3)$ are equal to $\frac{1}{2} \pi$; the Hessian matrix (31), however, is indefinite at this point which therefore cannot be a maximum. Finally, the last class of solutions obeys $\theta_{1}=\theta_{2}=\pi-\theta_{3}$. Therefore, one arrives at the conclusion that a configuration, which is a solution of Eqs. (17), (18) and (19) and corresponds to a local maximum of the probability density $(12)$, must satisfy $\left(\theta_{1}, \theta_{2}, \theta_{3}\right)=(\theta, \theta, \pi-\theta)$ together with $\left(\phi_{1}, \phi_{2}, \phi_{3}\right)=\left(0, \frac{4}{3} \pi, \frac{5}{3} \pi\right)$. The condition on the remaining angle $\theta$ is obtained from Eqs. (14), (15) and (16), which all reduce to the single equation

$$
3 \sin ^{2} \theta\left(\frac{3}{2} \sin ^{2} \theta-1\right)^{2}+2 \sin ^{2} \theta\left(\frac{3}{2} \sin ^{2} \theta-1\right)-\sin ^{2} \theta=0
$$

of which the non-trivial (i.e., $\theta \neq 0$ or $\pi$ ) solution is $\sin \theta=\frac{2 \sqrt{2}}{3}$. This implies therefore the existence of two extrema, namely

$$
\begin{aligned}
& \text { (a) : }\left(\theta_{1}, \theta_{2}, \theta_{3}\right) \approx(0.39 \pi, 0.39 \pi, 0.61 \pi), \quad\left(\phi_{1}, \phi_{2}, \phi_{3}\right)=\left(0, \frac{4 \pi}{3}, \frac{5 \pi}{3}\right), \\
& \text { (b) : }\left(\theta_{1}, \theta_{2}, \theta_{3}\right) \approx(0.61 \pi, 0.61 \pi, 0.39 \pi), \quad\left(\phi_{1}, \phi_{2}, \phi_{3}\right)=\left(0, \frac{4 \pi}{3}, \frac{5 \pi}{3}\right),
\end{aligned}
$$




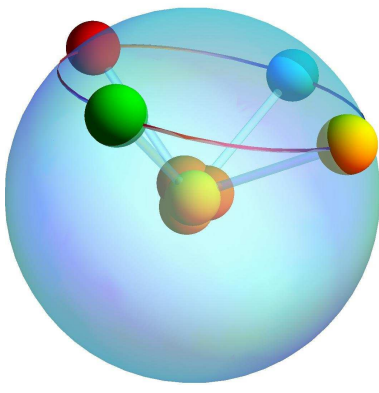

(a)

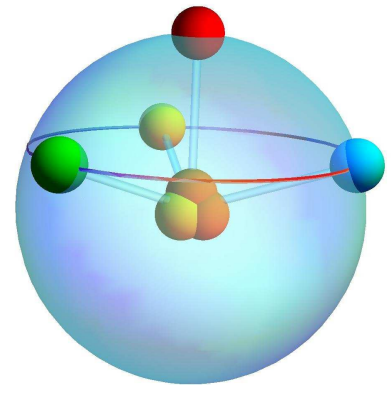

(b)

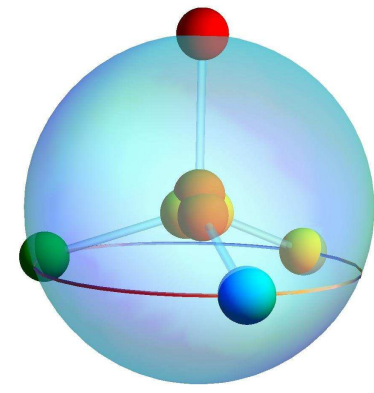

(c)

Fig. 3. The tetrahedral configurations that correspond to an absolute maximum of the probability density of the $\left|\left(p_{3 / 2}\right)^{4} ; 0^{+}\right\rangle$state. The configurations shown correspond to (a) $\left(\theta_{1}, \theta_{2}, \theta_{3}\right)=(0.39 \pi, 0.39 \pi, 0.61 \pi)$ and $\left(\phi_{1}, \phi_{2}, \phi_{3}\right)=\left(0, \frac{4}{3} \pi, \frac{5}{3} \pi\right)$, (b) $\left(\theta_{1}, \theta_{2}, \theta_{3}\right)=(0.61 \pi, 0.61 \pi, 0.39 \pi)$ and $\left(\phi_{1}, \phi_{2}, \phi_{3}\right)=\left(0, \frac{4}{3} \pi, \frac{5}{3} \pi\right)$, and (c) $\left(\theta_{1}, \theta_{2}, \theta_{3}\right)=(0.61 \pi, 0.61 \pi, 0.61 \pi)$ and $\left(\phi_{1}, \phi_{2}, \phi_{3}\right)=\left(\frac{1}{3} \pi, \pi, \frac{5}{3} \pi\right)$, always with $\theta_{4}=\phi_{4}=0$.

always with $\theta_{4}=\phi_{4}=0$. These configurations are shown in Figs. 3(a) and 3(b). After inversion of some of the particle positions in the configuration $3(\mathrm{a})$ or $3(\mathrm{~b})$, a third maximum is obtained of the form

$$
(\mathrm{c}):\left(\theta_{1}, \theta_{2}, \theta_{3}\right) \approx(0.61 \pi, 0.61 \pi, 0.61 \pi), \quad\left(\phi_{1}, \phi_{2}, \phi_{3}\right)=\left(\frac{\pi}{3}, \pi, \frac{5 \pi}{3}\right),
$$

the configuration of which is shown in Fig. 3(c). The Hessian matrix is negative definite in these points, which confirms that they are indeed local maxima.

In all three cases, not only the polar angles $\theta_{i}$ but also the relative angles $\theta_{i j}$ are either $0.61 \pi \simeq 109.47^{\circ}$, which is the tetrahedral angle in a regular tetrahedron, or $0.39 \pi \simeq 70.53^{\circ}$, the supplementary angle of the tetrahedral angle. Indeed, as can be seen in Fig. 3(c), the four particles are at the vertices of a regular tetrahedron and the other two configurations are derived from it; hence the name "tetrahedral configurations". Also, in all three cases, $\sin \theta_{i j}=$ $\frac{2 \sqrt{2}}{3}$ and the probability density (12) therefore equals $\frac{64}{27} c \simeq 2.37 c$, which is the largest value found so far. At the same time this shows that the greatcircle configurations (with probability density value $2 c$ ) correspond to local as opposed to absolute maxima.

It should not be forgotten, however, that the above solutions have been obtained by separating, for simplicity's sake, the three Eqs. (17), (18) and (19) into the set of equations given in Eqs. (20) and (21). It is therefore important to check whether all solutions have been found by this method, and this can be conveniently done by a Monte-Carlo simulation aimed at looking for the maximum in the probability density of the $\left|0_{1}^{+}\right\rangle$state. A random input is generated for each of the five variables in function (12) on the basis of a uniform distribution. The probability density is evaluated up to $10^{5}$ times for this set 
of random inputs and only the input which yields the maximum value for function (12) is retained. Running this simulation as many times as required, one always finds one of the three tetrahedral configurations in Fig. 3, which corroborates the earlier conclusion that these correspond to absolute maxima in the probability density of the $\left|0_{1}^{+}\right\rangle$state.

\section{The geometry of four identical particles in a $p$ shell}

In the previous section the geometry was determined of a specific four-particle state $\left|0_{1}^{+}\right\rangle \equiv\left|\left(p_{3 / 2}\right)^{4} ; 0^{+}\right\rangle$. In this section (Subsect. 3.1) this result is generalized to an arbitrary four-particle state in the $p$ shell, still assuming that the particles are identical. If the particles are coupled to angular momentum $J=0$, it is obvious that, besides the state considered previously, another one exists, namely $\left|0_{2}^{+}\right\rangle \equiv\left|\left(p_{3 / 2}\right)^{2}(0)\left(p_{1 / 2}\right)^{2}(0) ; 0^{+}\right\rangle$, that is, a state with one pair in $p_{3 / 2}$ and another in $p_{1 / 2}$, both coupled to angular momentum $J=0$. It has been claimed [6] that the nucleus ${ }^{8} \mathrm{He}$ in its ground state has $\left|0_{1}^{+}\right\rangle$as the dominant component but that $\left|0_{2}^{+}\right\rangle$represents a non-negligible fraction of it. In fact, the relative importance of these components in the ground state of ${ }^{8} \mathrm{He}$ depends on the magnitude of the spin-orbit splitting between the $0 p_{3 / 2}$ and $0 p_{1 / 2}$ orbits and on the mixing between these orbits due to the residual interaction. As a specific application, the results derived in Subsect. 3.1 therefore enable us to picture the geometry of the ground state of ${ }^{8} \mathrm{He}$ as a function of the spin-orbit splitting and the residual interaction. This is discussed in Subsect. 3.2.

\subsection{Angular probability density of a $\left|p^{4} ; L M_{L} S M_{S}\right\rangle$ state}

It is convenient to discuss the configurations of four particles in an $L S$-coupled basis for which there is a clear separation between the spatial coordinates and the spin (and, if applicable, the isospin) degrees of freedom. A four-particle state in a single- $\ell$ shell in $L S$ coupling can be written as

$$
\begin{aligned}
& \Psi_{\ell 4}\left[L^{\prime} S^{\prime}, L^{\prime \prime} S^{\prime \prime}\right] L M_{L} S M_{S} \\
& \propto \\
& \mathcal{A} \sum_{M_{L}^{\prime} M_{L}^{\prime \prime} m_{1} m_{2} m_{3} m_{4}}(1,2,3,4) \\
& \quad \times \sum_{M_{S}^{\prime} M_{S}^{\prime \prime} m_{1}^{\prime} m_{2}^{\prime} m_{3}^{\prime} m_{4}^{\prime}}^{\prime}\left(S^{\prime} M_{S}^{\prime} S^{\prime \prime} M_{S}^{\prime \prime} \mid S M_{S}\right)\left(s m_{1}^{\prime} s m_{2}^{\prime} \mid S^{\prime} M_{S}^{\prime}\right)\left(s m_{3}^{\prime} s m_{4}^{\prime} \mid S^{\prime \prime} M_{S}^{\prime \prime}\right) \\
& \quad \times Y_{\ell m_{1}}\left(\Omega_{1}\right) Y_{\ell m_{2}}\left(\Omega_{2}\right) Y_{\ell m_{3}}\left(\Omega_{3}\right) Y_{\ell m_{4}}\left(\Omega_{4}\right) \chi_{m_{1}^{\prime}}(1) \chi_{m_{2}^{\prime}}(2) \chi_{m_{3}^{\prime}}(3) \chi_{m_{4}^{\prime}}(4), \quad(36)
\end{aligned}
$$

with $s=1 / 2$ and where the operator $\mathcal{A}$ antisymmetrizes in the four particles. Since the radial part of the wave function is the same for the four particles, 
it can be put up front and can be omitted henceforth. In accordance with the theory of coefficients of fractional parentage (CFPs) [7], the intermediate angular momenta $\left(L^{\prime} S^{\prime}\right)$ and $\left(L^{\prime \prime} S^{\prime \prime}\right)$, resulting from the coupling of particles 1 and 2 , and 3 and 4 , respectively, form a (generally overcomplete) set of labels to characterize the antisymmetric four-particle state, besides its total orbital angular momentum $L$ and its total spin $S$, and their projections. An alternative way of writing the state is therefore

$$
\begin{aligned}
& \Psi_{\ell^{4}\left[L^{\prime} S^{\prime}, L^{\prime \prime} S^{\prime \prime}\right] L M_{L} S M_{S}}(1,2,3,4) \\
& \left.=\sum_{L_{a}^{\prime} S_{a}^{\prime} L_{a}^{\prime \prime} S_{a}^{\prime \prime}}\left[\ell^{2}\left(L_{a}^{\prime} S_{a}^{\prime}\right) \ell^{2}\left(L_{a}^{\prime \prime} S_{a}^{\prime \prime}\right) ; L S \mid\right\} \ell^{4}\left[L^{\prime} S^{\prime}, L^{\prime \prime} S^{\prime \prime}\right] L S\right] \\
& \quad \times\left[\Psi_{\ell^{2} L_{a}^{\prime} S_{a}^{\prime}}(1,2) \times \Psi_{\ell^{2} L_{a}^{\prime \prime} S_{a}^{\prime \prime}}(3,4)\right]_{M_{L} M_{S}}^{(L S)} .
\end{aligned}
$$

The normalized state $\Psi_{\ell^{2} L_{a}^{\prime} S_{a}^{\prime}}(1,2)$, antisymmetric in the first two particles, is coupled to the normalized state $\Psi_{\ell^{2} L_{a}^{\prime \prime} S_{a}^{\prime \prime}}(3,4)$, antisymmetric in the last two particles, to give a four-particle state with total quantum numbers $(L S)$ and their projections $\left(M_{L} M_{S}\right)$. A linear combination of such states, weighted with appropriate coefficients, yields a state which is antisymmetric in all four particles. The symbol $[\cdots \mid\} \cdots]$ is a CFP in $L S$ coupling, with the square brackets $\left[L^{\prime} S^{\prime}, L^{\prime \prime} S^{\prime \prime}\right]$ indicating the so-called parent state from which the state has been obtained after antisymmetrization. The CFPs appearing in Eq. (37) are known in closed form in terms of $9 j$ symbols and hence this expansion can be used for the analytic derivations given below. Alternatively, an explicit antisymmetrization of the expression (36), containing 24 permutations can be carried out. Care should then be taken to normalize the resulting antisymmetric state whereas the expansion (37) is normalized by definition.

The spatial probability density of the wave function (36) or (37) is an average over all magnetic substates $M_{L}$ and $M_{S}$,

$$
\begin{aligned}
& \mathcal{P}_{\ell^{4}\left[L^{\prime} S^{\prime}, L^{\prime \prime} S^{\prime \prime}\right] L S}\left(\bar{r}_{1}, \bar{r}_{2}, \bar{r}_{3}, \bar{r}_{4}\right)=\frac{1}{(2 L+1)(2 S+1)} \\
& \times \sum_{M_{L} M_{S}} \Psi_{\ell^{4}\left[L^{\prime} S^{\prime}, L^{\prime \prime} S^{\prime \prime}\right] L M_{L} S M_{S}}^{*}(1,2,3,4) \Psi_{\ell^{4}\left[L^{\prime} S^{\prime}, L^{\prime \prime} S^{\prime \prime}\right] L M_{L} S M_{S}}(1,2,3,4),
\end{aligned}
$$

where it is understood that the spin degrees of freedom have been integrated out with use of

$$
\chi_{m_{i}^{\prime}}^{*}(i) \chi_{m_{i}^{\prime \prime}}(i)=\delta_{m_{i}^{\prime} m_{i}^{\prime \prime}}, \quad i=1,2,3,4 .
$$

For four particles in a single- $\ell$ shell, the radial dependence can be factored out of the spatial probability density, and one is left with an angular probability density $\mathcal{P}_{\ell^{4}\left[L^{\prime} S^{\prime}, L^{\prime \prime} S^{\prime \prime}\right] L S}\left(\Omega_{1}, \Omega_{2}, \Omega_{3}, \Omega_{4}\right)$. Since the spherical harmonic $Y_{\ell m^{\prime}}(\Omega)$ 
can be written as

$$
Y_{\ell m^{\prime}}(\Omega)=\sum_{\substack{r^{\prime}=0 \\ \text { even }}}^{\ell} a_{\ell m^{\prime}}^{r^{\prime}}(\cos \theta)^{\ell-m^{\prime}-r^{\prime}}(\sin \theta)^{m^{\prime}}(\cos \phi+i \sin \phi)^{m^{\prime}}
$$

in terms of certain coefficients $a_{\ell m^{\prime}}^{r^{\prime}}$, the angular probability density for four particles acquires the generic form

$$
\begin{aligned}
& \mathcal{P}_{\ell^{4}\left[L^{\prime} S^{\prime}, L^{\prime \prime} S^{\prime \prime}\right] L S}\left(\Omega_{1}, \Omega_{2}, \Omega_{3}, \Omega_{4}\right) \\
& =\sum_{\bar{r} \bar{s}}\left(\prod_{j=1}^{4}\left(\cos \theta_{j}\right)^{2 \ell-r_{j}-s_{j}}\left(\sin \theta_{j}\right)^{s_{j}}\right)\left(\sum_{\bar{n}} a_{\bar{r} \bar{s} \bar{n}} \prod_{i=1}^{4}\left(\cos \phi_{i}\right)^{n_{i}}\left(\sin \phi_{i}\right)^{s_{i}-n_{i}}\right),
\end{aligned}
$$

where $\bar{r}, \bar{s}$ and $\bar{n}$ are short-hand notations for sets of four integers $\left\{r_{1} r_{2} r_{3} r_{4}\right\}$, $\left\{s_{1} s_{2} s_{3} s_{4}\right\}$ and $\left\{n_{1} n_{2} n_{3} n_{4}\right\}$, respectively, which satisfy the restrictions $0 \leq$ $r_{i} \leq 2 \ell$ and $r_{i}$ must be even, $0 \leq s_{i} \leq 2 \ell-r_{i}$ and $0 \leq n_{i} \leq s_{i}$. Furthermore, for notational simplicity the dependence on the state's labels $\ell^{4}\left[L^{\prime} S^{\prime}, L^{\prime \prime} S^{\prime \prime}\right] L S$ is suppressed in the coefficients $a_{\bar{r} \bar{s} \bar{n}}$.

For $\ell=1$ (the $p$ shell) the spherical harmonics (4) lead to an angular probability density of the form

$$
\begin{aligned}
& \mathcal{P}_{p^{4} L S}\left(\Omega_{1}, \Omega_{2}, \Omega_{3}, \Omega_{4}\right) \\
& =\sum_{\bar{s}}\left(\prod_{j=1}^{4}\left(\cos \theta_{j}\right)^{2-s_{j}}\left(\sin \theta_{j}\right)^{s_{j}}\right)\left(\sum_{\bar{n}} a_{\bar{s} \bar{n}} \prod_{i=1}^{4}\left(\cos \phi_{i}\right)^{n_{i}}\left(\sin \phi_{i}\right)^{s_{i}-n_{i}}\right) .
\end{aligned}
$$

A state of four identical particles in the $p$ shell is completely specified by its total orbital angular momentum $L$ and its total spin $S$, and no intermediate labels $\left[L^{\prime} S^{\prime}, L^{\prime \prime} S^{\prime \prime}\right]$ are needed. For $\ell=1$ all labels $r_{i}$ must be zero [see Eq. (40)] and the sum over $\bar{r}$ can be omitted. Furthermore, for the $p$ shell the possible sets for $\bar{s}$ are: $\{2222\}(1),\{2211\}(6),\{2220\}(4),\{1111\}(1),\{2200\}(6)$, $\{2110\}(12),\{2000\}(4),\{1100\}$ (6) and $\{0000\}(1)$, where the number in brackets after each set indicates the number of possible permutations. For example, the six permutations associated with $\{2211\}$ are: $\{2211\},\{2121\}$, $\{2112\},\{1221\},\{1212\}$ and $\{1122\}$. Thus, the sum over $\bar{s}$ contains in fact 41 terms.

The question is now whether the expression (41) can be converted to one which depends on the relative angles $\theta_{i j}$ only. This, of course, is not always possible for general coefficients $a_{\bar{r} \bar{s} \bar{n}}$ but, from symmetry arguments, it should be possible to do so for the $a_{\bar{r} \bar{s} \bar{n}}$ associated with an angular probability density. In Appendix B an algorithm is given, based on trigonometric identities, for the symbolic reduction of the angular probability density (41) into an expression depending on $\cos \theta_{i j}$ only. 
Table 1

The coefficients $a_{L S}, b_{L S}, c_{L S}$ and $d_{L S}$ in the angular probability density (43) of four identical particles in the $p$ shell.

\begin{tabular}{cccccc}
\hline$L$ & $S$ & $a_{L S}$ & $b_{L S}$ & $c_{L S}$ & $d_{L S}$ \\
\hline 0 & 0 & - & - & $\frac{9}{512 \pi^{4}}$ & $-\frac{9}{512 \pi^{4}}$ \\
1 & 1 & $\frac{27}{2048 \pi^{4}}$ & $-\frac{9}{2048 \pi^{4}}$ & $-\frac{9}{2048 \pi^{4}}$ & $\frac{9}{1024 \pi^{4}}$ \\
2 & 0 & $\frac{81}{10240 \pi^{4}}$ & $-\frac{27}{10240 \pi^{4}}$ & $\frac{9}{2048 \pi^{4}}$ & $-\frac{9}{5120 \pi^{4}}$ \\
\hline
\end{tabular}

Application of the algorithm of Appendix B to the angular probability density (42) of four particles in the $p$ shell leads to the following expression of this quantity in terms of the relative angles $\theta_{i j}$ :

$$
\begin{aligned}
& \mathcal{P}_{p^{4} ; L S}\left(\theta_{12}, \theta_{13}, \theta_{14}, \theta_{23}, \theta_{24}, \theta_{34}\right) \\
& =a_{L S}+b_{L S} \sum_{(i j)} \cos ^{2} \theta_{i j}+c_{L S} \sum_{(i j) \neq(k l)} \cos ^{2} \theta_{i j} \cos ^{2} \theta_{k l} \\
& \quad+d_{L S} \sum_{(i j) \neq(k l) \neq(m n) \neq(p q)} \cos \theta_{i j} \cos \theta_{k l} \cos \theta_{m n} \cos \theta_{p q}
\end{aligned}
$$

where the summations are over all possible pairs $(i j),(k l), \ldots$ with $i \neq j$, $k \neq l, \ldots$, such that a particle index occurs at most twice in each term of the sum. Furthermore, if the summation is over several pairs, they should be distinct. Therefore, the first summation contains six terms [(12), (13), (14), (23), (24) and (34)] and the second and third summation have three terms each $[(12)(34),(13)(24)$ and $(14)(23)$, and $(12)(13)(24)(34),(12)(14)(23)(34)$ and $(13)(14)(23)(24)$, respectively]. The coefficients $a_{L S}, b_{L S}, c_{L S}$ and $d_{L S}$ are determined by the geometry of the $p$ shell and are given in Table 1 .

In the case of two particles, the angular probability density was written as a sum over Legendre polynomials in the cosine of the relative angle $\theta_{i j}$ [see Eq. (23) of I]. The expression (43) is a generalization of this result to four particles. Equation (43) is valid for the $p$ shell but, since the algorithm of Appendix B can be applied to any shell, the present results constitute a generalization of the angular probability density in terms of relative angles from two to four particles.

For the states with $L=S$ the $p$-shell results can be further simplified. For $L=S=0$, one finds ${ }^{3}$

$$
\mathcal{P}_{p^{4} ; 00}=\frac{9}{1024 \pi^{4}} \sum_{(i j) \neq(k l)}\left(\cos \theta_{i k} \cos \theta_{j l}-\cos \theta_{i l} \cos \theta_{j k}\right)^{2}
$$

$\overline{3}$ For notational simplicity the arguments $\theta_{i j}$ in the angular probability densities $\mathcal{P}_{\alpha}$ are suppressed in the following. 


$$
=\frac{9}{1024 \pi^{4}} \sum_{(i j) \neq(k l)} \sin ^{2} \theta_{i j} \sin ^{2} \theta_{k l} \cos ^{2} \theta_{i j, k l},
$$

where use is made of an identity which relates the angles $\theta_{i j}$ to $\theta_{i j, k l}$ (the angle between $\bar{r}_{i j}$ and $\bar{r}_{k l}$ ),

$$
\sin \theta_{i j} \sin \theta_{k l} \cos \theta_{i j, k l}=\cos \theta_{i k} \cos \theta_{j l}-\cos \theta_{j k} \cos \theta_{i l}
$$

Similarly, for $L=S=1$, one finds from the coefficients given in Table 1 the following expression:

$$
\mathcal{P}_{p^{4} ; 11}=\frac{9}{2048 \pi^{4}} \sum_{(i j) \neq(k l)} \sin ^{2} \theta_{i j} \sin ^{2} \theta_{k l} \sin ^{2} \theta_{i j, k l} .
$$

The angular probability density of a state with $J=0$ (which necessarily must be a linear combination of the two states $L=0, S=0$ and $L=1, S=1$ ) can therefore be expressed as a three-termed sum over pairs of particles $(i j)(k l)$ with $(i j)(k l)=(12)(34),(13)(24)$ and $(14)(23)$.

For $J=0$ these results can be converted to the more familiar $j j$-coupled basis by noting that the states $\left|0_{1}^{+}\right\rangle$and $\left|0_{2}^{+}\right\rangle$can be written as linear combinations of the states with $L=0, S=0$ and $L=1, S=1$. Consequently, the angular probability densities of the $j j$-coupled states are

$$
\mathcal{P}_{0_{1}^{+}}=\frac{1}{3} \mathcal{P}_{p^{4} ; 00}+\frac{2}{3} \mathcal{P}_{p^{4} ; 11}, \quad \mathcal{P}_{0_{2}^{+}}=\frac{2}{3} \mathcal{P}_{p^{4} ; 00}+\frac{1}{3} \mathcal{P}_{p^{4} ; 11}
$$

This leads to the expressions

$$
\mathcal{P}_{0_{1}^{+}}=\frac{3}{1024 \pi^{4}} \sum_{(i j) \neq(k l)} \sin ^{2} \theta_{i j} \sin ^{2} \theta_{k l}
$$

which coincides with the result (11), and

$$
\mathcal{P}_{0_{2}^{+}}=\frac{3}{2048 \pi^{4}} \sum_{(i j) \neq(k l)} \sin ^{2} \theta_{i j} \sin ^{2} \theta_{k l}\left(1+3 \cos ^{2} \theta_{i j, k l}\right),
$$

a result which can also be derived in cartesian coordinates.

A study of the angular probability density (49), in the same way as is done in Subsect. 2.2 for the $\left|0_{1}^{+}\right\rangle$state, reveals the same geometric configurations for the $\left|0_{2}^{+}\right\rangle$state, the only difference being that the great-circle configurations are the absolute maxima in this case with the value $4 c$ while the tetrahedral configurations are local maxima with the value $\frac{32}{27} c$. 


\subsection{The geometry of the ground state of ${ }^{8} \mathrm{He}$}

Under the assumption of the confinement of the four outer neutrons in ${ }^{8} \mathrm{He}$ to the $0 p$ shell, its ground state $\left|0_{\mathrm{gs}}^{+}\right\rangle$can be written as the linear combination

$$
\left|0_{\mathrm{gs}}^{+}\right\rangle=\alpha\left|(0 p)^{4} ; L=0, S=0\right\rangle+\beta\left|(0 p)^{4} ; L=1, S=1\right\rangle
$$

with $\alpha^{2}+\beta^{2}=1$. How the angular probability density as well as its maxima evolve with the mixing of the two states is studied in this subsection.

With the expressions derived earlier it is a simple matter to find the angular probability density for the admixed state (50),

$$
\begin{aligned}
\mathcal{P}_{0_{\mathrm{gs}}^{+}} & =(1-\eta) \mathcal{P}_{p^{4} ; 00}+\eta \mathcal{P}_{p^{4} ; 11} \\
& =\frac{9}{2048 \pi^{4}} \sum_{(i j) \neq(k l)} \sin ^{2} \theta_{i j} \sin ^{2} \theta_{k l}\left[\eta+(2-3 \eta) \cos ^{2} \theta_{i j, k l}\right],
\end{aligned}
$$

where $1-\eta=\alpha^{2}$ and $\eta=\beta^{2}$, so that $\eta$ varies between 0 and 1 . The properties of $\mathcal{P}_{0_{\mathrm{gs}}^{+}}$depend on the parameter $\eta$, which controls the competition between the terms $\sin ^{2} \theta_{i j} \sin ^{2} \theta_{k l}$ which favor the tetrahedral configurations and the terms $\sin ^{2} \theta_{i j} \sin ^{2} \theta_{k l} \cos ^{2} \theta_{i j, k l}$ which favor the great-circle configurations.

Obviously, for $\eta=0$ the angular probability density associated with the $\left|(0 p)^{4} ; L=0, S=0\right\rangle$ state is recovered while $\eta=1$ corresponds to $\mid(0 p)^{4} ; L=$ $1, S=1\rangle$. The $j j$-coupled states $\left|0_{1}^{+}\right\rangle$and $\left|0_{2}^{+}\right\rangle$are obtained for $\eta=\frac{2}{3}$ and $\eta=\frac{1}{3}$, respectively. The critical points where the probability density peaks (locally or globally) for the $\left|0_{1}^{+}\right\rangle$state (see Subsect. 2.2), also have vanishing first partial derivatives for an arbitrary admixed state. They are thus good candidates for maxima and it is therefore natural to examine the character of the great-circle and tetrahedral configurations in admixed states.

For any of the great-circle configurations, the angular probability density $\mathcal{P}_{0_{\mathrm{gs}}^{+}}$ has the value

$$
\mathcal{P}_{0_{\mathrm{gs}}^{+}}^{\mathrm{gcc}}=\frac{9}{512 \pi^{4}}(1-\eta)=6(1-\eta) c,
$$

where $c$ is the constant introduced earlier, $c=3 \cdot 2^{-10} \pi^{-4}$. To determine the nature of the angular probability density at this point (i.e., maximum, minimum or saddle point), the Hessian matrix needs to be constructed for general $\eta$. Take as an example the configuration $\left(\theta_{1}, \theta_{2}, \theta_{3}\right)=\left(\frac{1}{2} \pi, \frac{1}{2} \pi, 0\right)$ and $\left(\phi_{1}, \phi_{2}, \phi_{3}\right)=(0,0,0)$ [see Fig. 1(a)]. The Hessian matrix at this point takes 


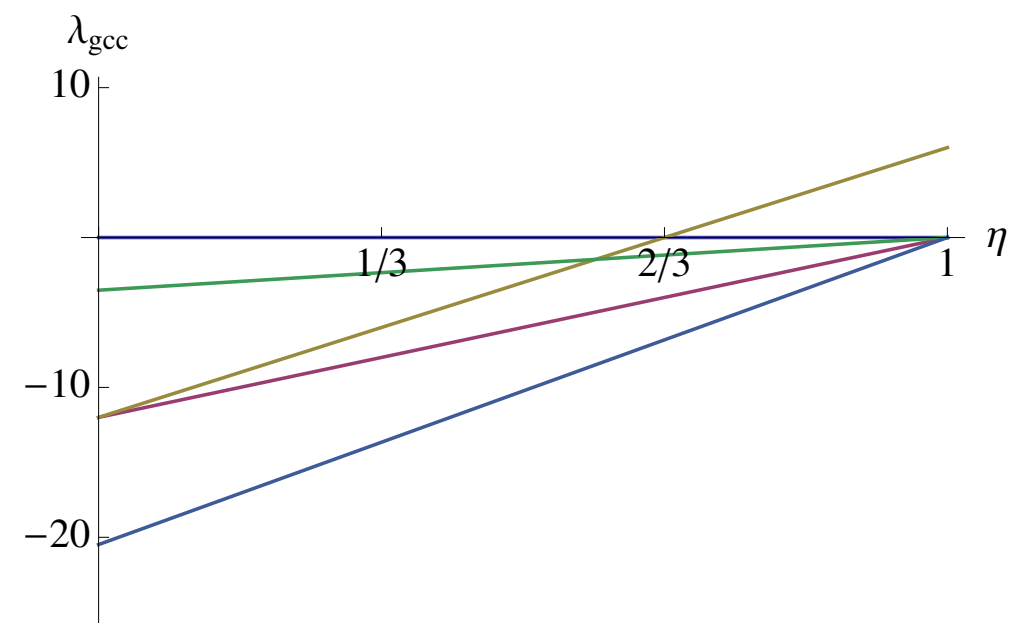

Fig. 4. The eigenvalues of the Hessian matrix at any of the great-circle configurations. The eigenvalues are expressed in units $c=3 \cdot 2^{-10} \pi^{-4}$.

the form

$$
c\left(\begin{array}{ccccc}
-12 \eta_{1,-1} & 0 & 6 \eta_{1,-1} & 0 & 0 \\
0 & -12 \eta_{1,-1} & 6 \eta_{1,-1} & 0 & 0 \\
6 \eta_{1,-1} & 6 \eta_{1,-1} & -12 \eta_{1,-1} & 0 & 0 \\
0 & 0 & 0 & -6 \eta_{2,-3} & 0 \\
0 & 0 & 0 & 0 & 0
\end{array}\right)
$$

where $\eta_{k, l} \equiv k+l \eta$. The upper $3 \times 3$ part of the matrix has the eigenvalues $-12(1-\eta) c$ and $-6(2 \pm \sqrt{2})(1-\eta) c$ which are all negative for $0 \leq \eta<1$. The character of the critical point is determined by the remaining eigenvalues, 0 and $-6(2-3 \eta) c$. Because of the former, the Hessian matrix is never negative definite; the latter eigenvalue changes sign at $\eta=\frac{2}{3}$.

The same eigenvalues are obtained for the other great-circle configurations and they are plotted in Fig. 4. In Subsect. 2.2 it is shown that at $\eta=\frac{2}{3}$ (i.e., for the $\left|0_{1}^{+}\right\rangle$state) the great-circle configurations are local maxima. For $\eta>\frac{2}{3}$, the Hessian matrix is indefinite, indicating that the great-circle configurations no longer correspond to a maximum. For $\eta<\frac{2}{3}$, the Hessian matrix is always negative semi-definite with one vanishing eigenvalue. Yet, since the submatrix associated with the second derivatives with respect to $\theta_{i}$ is negative definite, one concludes, as in Subsect. 2.2, that any of the great-circle configurations yields an improper (either local or global) maximum of the angular probability density.

For any of the tetrahedral configurations, the angular probability density $\mathcal{P}_{0_{\mathrm{gs}}^{+}}$ 


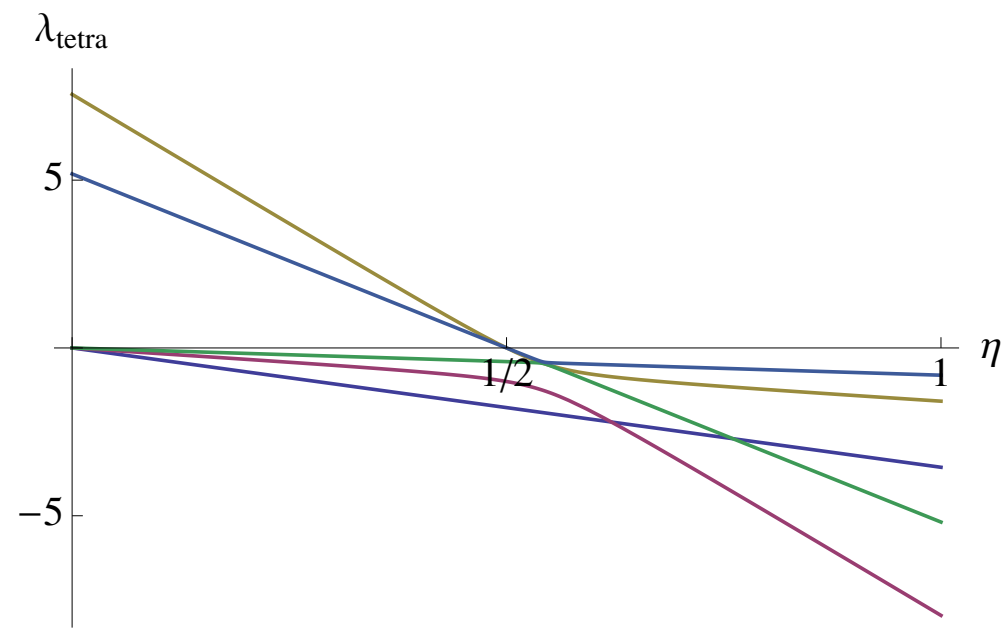

Fig. 5. The eigenvalues of the Hessian matrix at any of the tetrahedral configurations. The eigenvalues are expressed in units $c=3 \cdot 2^{-10} \pi^{-4}$.

has the value

$$
\mathcal{P}_{0_{\mathrm{gs}}^{+}}^{\text {tetra }}=\frac{1}{96 \pi^{4}} \eta=\frac{32}{9} \eta c .
$$

To examine the character of the extremum at these configurations, take as an example the configuration $\left(\theta_{1}, \theta_{2}, \theta_{3}\right)=(0.39 \pi, 0.39 \pi, 0.61 \pi)$ and $\left(\phi_{1}, \phi_{2}, \phi_{3}\right)=$ $\left(0, \frac{4}{3} \pi, \frac{5}{3} \pi\right)$ [see Fig. 3(a)]. The Hessian matrix at this point takes the form

$$
c\left(\begin{array}{ccccc}
\frac{4}{3} \eta_{2,-5} & -\frac{2}{9} \eta_{6,7} & \frac{2}{9} \eta_{6,7} & -\frac{4}{9} \sqrt{\frac{2}{3}} \eta_{6,-11} & \frac{4}{9} \sqrt{\frac{2}{3}} \eta_{6,-11} \\
-\frac{2}{9} \eta_{6,7} & \frac{4}{3} \eta_{2,-5} & \frac{2}{9} \eta_{6,7} & 0 & -\frac{4}{9} \sqrt{\frac{2}{3}} \eta_{6,-11} \\
\frac{2}{9} \eta_{6,7} & \frac{2}{9} \eta_{6,7} & \frac{4}{3} \eta_{2,-5} & -\frac{4}{9} \sqrt{\frac{2}{3}} \eta_{6,-11} & 0 \\
-\frac{4}{9} \sqrt{\frac{2}{3}} \eta_{6,-11} & 0 & -\frac{4}{9} \sqrt{\frac{2}{3}} \eta_{6,-11} & \frac{32}{27} \eta_{2,-5} & -\frac{16}{27} \eta_{2,-5} \\
\frac{4}{9} \sqrt{\frac{2}{3}} \eta_{6,-11} & -\frac{4}{9} \sqrt{\frac{2}{3}} \eta_{6,-11} & 0 & -\frac{16}{27} \eta_{2,-5} & \frac{32}{27} \eta_{2,-5}
\end{array}\right) .
$$

This matrix has the eigenvalues

$$
\begin{aligned}
& -\frac{32}{9} \eta c, \\
& \frac{1}{9}\left(34-77 \eta \pm \sqrt{1156-4212 \eta+3881 \eta^{2}}\right) c, \\
& \frac{1}{27}\left(70-151 \eta \pm \sqrt{4900-18068 \eta+16657 \eta^{2}}\right) c .
\end{aligned}
$$

The same eigenvalues are obtained for the other tetrahedral configurations and they are plotted in Fig. 5. Two eigenvalues vanish at $\eta=\frac{1}{2}$. For $\eta>\frac{1}{2}$, all the eigenvalues are negative, the Hessian matrix is negative definite and the tetrahedral configurations are maxima. For $\eta<\frac{1}{2}$, some of the eigenvalues 


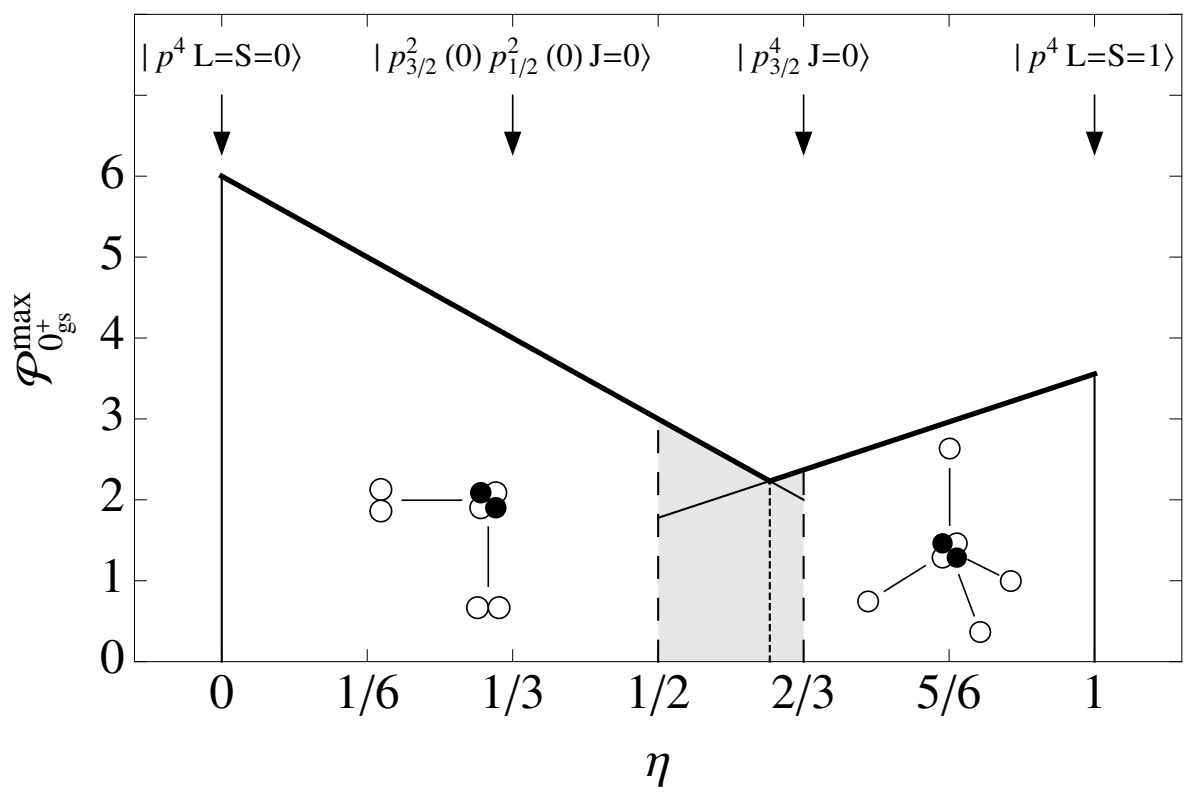

Fig. 6. The evolution of the value of the angular probability density at its maximum associated with the $\left|0_{\mathrm{gs}}^{+}\right\rangle$ground state of four identical particles in the $p$ shell, as a function of the parameter $\eta$. The region $0 \leq \eta \leq \frac{27}{43}$ has great-circle configurations as global maxima while in the zone $\frac{27}{43} \leq \eta \leq 1$ the angular probability density associated with the tetrahedral configurations attains a global maximum. The boundaries of existence of local maxima are indicated with the dashed lines and the coexistence region where the angular probability density peaks for the great-circle and for the tetrahedral configurations is shown in gray. The angular probability density is expressed in units $c=3 \cdot 2^{-10} \pi^{-4}$.

are positive and some are negative, that is, the matrix is indefinite which is indicative of a saddle point. Left unsolved is the tricky point $\eta=\frac{1}{2}$ itself where two of the eigenvalues of the Hessian matrix are zero. The splitting lemma of Thom's catastrophe theory [8] provides a way of transforming a function in a neighborhood of a degenerate critical point (i.e., a critical point where the determinant of the Hessian matrix vanishes) to a canonical form through a series of changes of variables. The local properties at the degenerate critical point can then be studied via the catastrophe germ that is left over in the Taylor expansion of the function after the transformation. An analysis of this type shows that the catastrophe germ is cubic at $\eta=\frac{1}{2}$ and that a tetrahedral configuration is not a local maximum at this point. One concludes that for $\eta>\frac{1}{2}$ tetrahedral configurations are (local or global) maxima but they are no longer for $\eta \leq \frac{1}{2}$.

The preceding results can be summarized with Fig. 6 which shows a diagram for the geometry of four identical particles in the $p$ shell. The control parameter $\eta$ along the $x$ axis is related to the structure of the ground state and incorporates information on the spin-orbit splitting and the character of the 
residual interaction. As a function of $\eta$ is plotted the maximum value of the angular probability density which varies between $6 c$ and $\frac{92}{43} c$. To the right of the dashed line at $\eta=\frac{1}{2}$ (excluding $\frac{1}{2}$ ), the tetrahedral configurations are local maxima; to the right of the dotted line at $\eta=\frac{27}{43}$ is the region where the tetrahedral configurations are absolute maxima. The great-circle configurations are maxima to the left of the dashed line $\eta=\frac{2}{3}$ (including $\frac{2}{3}$ ), and absolute maxima in the zone $\eta \leq \frac{27}{43}$. In the language of phase transitions, the area in gray can be viewed as a region of coexistence where the angular probability density peaks for the great-circle and for the tetrahedral configurations. This area is bounded by the spinodal points $\eta=\frac{1}{2}$ and $\eta=\frac{2}{3}$. It is separated in two distinct regions by the Maxwell point $\eta=\frac{27}{43}$ where the maximum values of the angular probability density are the same for the two configurations.

It should be pointed out that this analysis does not differentiate between the three great-circle configurations nor between the three tetrahedral configurations, discussed in Subsect. 2.2. In other words, the diagram in Fig. 6 is valid for any of the three great-circle configurations and for any of the three tetrahedral configurations. This is a property of the $p$ shell akin to the equiprobability of the di-neutron and cigar-like configurations if a pure $p$-shell configuration is adopted for two particles. As was shown in part I, admixtures from higher shells lead to a predominance of the di-neutron over the cigar-like configuration. It would be of interest to study similarly the effect of excitations into higher shells on the spatial correlations of a four-particle system and, specifically, to determine which of the three great-circle configurations becomes the dominant one as a result of such excitations.

While one cannot at this point select a favorite among the three great-circle or the three tetrahedral configurations, the present results indicate that the former rather than the latter yield a more accurate intuitive picture of the geometry of ${ }^{8} \mathrm{He}$. Although the spin-orbit splitting between the $0 p_{3 / 2}$ and the $0 p_{1 / 2}$ orbits favors the formation of a $\left|\left(0 p_{3 / 2}\right)^{4} ; 0^{+}\right\rangle$ground state in ${ }^{8} \mathrm{He}$, this tendency is counterbalanced by the short-range, attractive character of the residual interaction which lowers the energy of states with spatial symmetry and hence prefers a ground state with $L=S=0$. Empirical shell-model interactions, fitted to the observed spectroscopy of $0 p$-shell nuclei, arguably are the best way to decide on the outcome of this competition. For example, the CohenKurath interaction [9] yields a ground state for ${ }^{8} \mathrm{He}$ which is predominantly spatially symmetric with only a small admixture of the $\left|(0 p)^{4} ; L=S=1\right\rangle$ state, $\beta^{2}=\eta=0.01$. For such a small value of $\eta$, the appropriate geometry is one of the three great-circle configurations. 


\section{Conclusions}

In part I of this series, the existence of the two well-known geometric configurations of ${ }^{6} \mathrm{He}$, commonly referred to as di-neutron and cigar-like, were shown to be an inescapable consequence of the geometry of the $0 p$ shell. In this paper, with the example of the halo nucleus ${ }^{8} \mathrm{He}$ in mind, this geometric view of the shell model was extended from two to four particles. It was found that a fruitful way to approach this problem consists of converting the probability density of an arbitrary four-particle state into an expression that depends on the six angles $\theta_{i j}$ between pairs of position vectors of the particles $i$ and $j$. This conversion was achieved explicitly for four identical particles in a $p$ shell on the basis of an algorithm valid for any shell. This work, therefore, extends the knowledge of the angular correlation function, which was established for two particles, to four particles.

While two configurations, di-neutron and cigar-like, arise naturally for two particles in a $p$ shell, a system of four identical particles exhibits a richer geometry. Six favored four-particle configurations are found in the $p$ shell which fall into two classes, great-circle and tetrahedral, with three members each. In each class the three configurations are equiprobable as long as the particles are confined to the $p$ shell. Whether the great-circle or the tetrahedral configurations are dominant depends on the dynamics of the problem and, specifically, on the competition between the spin-orbit splitting and the strength of the short-range residual interaction. This work, therefore, establishes a "phase diagram" for the geometry of four particles in the $p$ shell. In addition, it appears from the present study that, for a realistic interaction in the $p$ shell, greatcircle configurations are favored. But it remains to be studied which of the three great-circle configurations will turn out dominant if excitations out of the $p$ shell are allowed.

The present study was motivated by the ongoing experimental work on ${ }^{8} \mathrm{He}$ and was therefore concerned with four identical particles in a $p$ shell. An obvious generalization is to attempt to construct similar geometries for three particles and/or for different shells. Nuclear examples of such systems do not spring to mind but we believe that theoretical studies of this kind have an intrinsic interest by themselves. Of more immediate relevance to nuclear structure would be the generalization of the present formalism to include isospin, which, probably, can be achieved without too much effort for particles in the $p$ shell. A study of this kind would provide us with information on the geometric structure of mixed systems of neutrons and protons, and possibly on the role played by neutron-neutron, neutron-proton and proton-proton pairs in such systems. 


\section{Acknowledgments}

We wish to thank Navin Alahari, Kris Heyde and Peter Schuck for fruitful discussions at various stages of this work. This work was partially supported by the Agence Nationale de Recherche, France, under contract ANR-07-BLAN0256-03.

\section{A The angular probability density in relative angles $\theta_{i j}$. Derivation in cartesian coordinates}

In this appendix we express the angular probability density associated with the $\left|0_{1}^{+}\right\rangle \equiv\left|\left(p_{3 / 2}\right)^{4} ; 0^{+}\right\rangle$state with the wave function (6), in terms of the relative angles $\theta_{i j}$. Use is made of cartesian coordinates. The starting point is the sum (6) in which the spatial dependence is specified by the following expressions:

$$
\begin{aligned}
& \Phi_{--++}\left(\bar{r}_{1}, \bar{r}_{2}, \bar{r}_{3}, \bar{r}_{4}\right)=\Phi_{++--}^{*}\left(\bar{r}_{1}, \bar{r}_{2}, \bar{r}_{3}, \bar{r}_{4}\right) \\
& =x_{2} x_{4} y_{1} y_{3}-x_{1} x_{4} y_{2} y_{3}-x_{2} x_{3} y_{1} y_{4}+x_{1} x_{3} y_{2} y_{4}+x_{2} x_{4} z_{1} z_{3}+y_{2} y_{4} z_{1} z_{3} \\
& -x_{1} x_{4} z_{2} z_{3}-y_{1} y_{4} z_{2} z_{3}-x_{2} x_{3} z_{1} z_{4}-y_{2} y_{3} z_{1} z_{4}+x_{1} x_{3} z_{2} z_{4}+y_{1} y_{3} z_{2} z_{4} \\
& -i\left(x_{4} y_{2} z_{1} z_{3}-x_{2} y_{4} z_{1} z_{3}-x_{4} y_{1} z_{2} z_{3}+x_{1} y_{4} z_{2} z_{3}-x_{3} y_{2} z_{1} z_{4}+x_{2} y_{3} z_{1} z_{4}\right. \\
& \left.+x_{3} y_{1} z_{2} z_{4}-x_{1} y_{3} z_{2} z_{4}\right) \text {, } \\
& \Phi_{-+-+}\left(\bar{r}_{1}, \bar{r}_{2}, \bar{r}_{3}, \bar{r}_{4}\right)=\Phi_{+-+-}^{*}\left(\bar{r}_{1}, \bar{r}_{2}, \bar{r}_{3}, \bar{r}_{4}\right) \\
& =-x_{3} x_{4} y_{1} y_{2}+x_{1} x_{4} y_{2} y_{3}+x_{2} x_{3} y_{1} y_{4}-x_{1} x_{2} y_{3} y_{4}-x_{3} x_{4} z_{1} z_{2}-y_{3} y_{4} z_{1} z_{2} \\
& +x_{1} x_{4} z_{2} z_{3}+y_{1} y_{4} z_{2} z_{3}+x_{2} x_{3} z_{1} z_{4}+y_{2} y_{3} z_{1} z_{4}-x_{1} x_{2} z_{3} z_{4}-y_{1} y_{2} z_{3} z_{4} \\
& +i\left(x_{4} y_{3} z_{1} z_{2}-x_{3} y_{4} z_{1} z_{2}-x_{4} y_{1} z_{2} z_{3}+x_{1} y_{4} z_{2} z_{3}+x_{3} y_{2} z_{1} z_{4}-x_{2} y_{3} z_{1} z_{4}\right. \\
& \left.+x_{2} y_{1} z_{3} z_{4}-x_{1} y_{2} z_{3} z_{4}\right) \\
& \Phi_{-++-}\left(\bar{r}_{1}, \bar{r}_{2}, \bar{r}_{3}, \bar{r}_{4}\right)=\Phi_{+--+}^{*}\left(\bar{r}_{1}, \bar{r}_{2}, \bar{r}_{3}, \bar{r}_{4}\right) \\
& =x_{3} x_{4} y_{1} y_{2}-x_{2} x_{4} y_{1} y_{3}-x_{1} x_{3} y_{2} y_{4}+x_{1} x_{2} y_{3} y_{4}+x_{3} x_{4} z_{1} z_{2}+y_{3} y_{4} z_{1} z_{2} \\
& -x_{2} x_{4} z_{1} z_{3}-y_{2} y_{4} z_{1} z_{3}-x_{1} x_{3} z_{2} z_{4}-y_{1} y_{3} z_{2} z_{4}+x_{1} x_{2} z_{3} z_{4}+y_{1} y_{2} z_{3} z_{4} \\
& +i\left(x_{4} y_{3} z_{1} z_{2}-x_{3} y_{4} z_{1} z_{2}-x_{4} y_{2} z_{1} z_{3}+x_{2} y_{4} z_{1} z_{3}+x_{3} y_{1} z_{2} z_{4}-x_{1} y_{3} z_{2} z_{4}\right. \\
& \left.-x_{2} y_{1} z_{3} z_{4}+x_{1} y_{2} z_{3} z_{4}\right), \\
& \Phi_{---+}\left(\bar{r}_{1}, \bar{r}_{2}, \bar{r}_{3}, \bar{r}_{4}\right)=-\Phi_{+++-}^{*}\left(\bar{r}_{1}, \bar{r}_{2}, \bar{r}_{3}, \bar{r}_{4}\right) \\
& =-x_{3} y_{2} y_{4} z_{1}+x_{2} y_{3} y_{4} z_{1}+x_{3} y_{1} y_{4} z_{2}-x_{1} y_{3} y_{4} z_{2}-x_{2} y_{1} y_{4} z_{3}+x_{1} y_{2} y_{4} z_{3} \\
& +i\left(x_{3} x_{4} y_{2} z_{1}-x_{2} x_{4} y_{3} z_{1}-x_{3} x_{4} y_{1} z_{2}+x_{1} x_{4} y_{3} z_{2}+x_{2} x_{4} y_{1} z_{3}-x_{1} x_{4} y_{2} z_{3}\right), \\
& \Phi_{--+-}\left(\bar{r}_{1}, \bar{r}_{2}, \bar{r}_{3}, \bar{r}_{4}\right)=-\Phi_{++-+}^{*}\left(\bar{r}_{1}, \bar{r}_{2}, \bar{r}_{3}, \bar{r}_{4}\right) \\
& =x_{4} y_{2} y_{3} z_{1}-x_{2} y_{3} y_{4} z_{1}-x_{4} y_{1} y_{3} z_{2}+x_{1} y_{3} y_{4} z_{2}+x_{2} y_{1} y_{3} z_{4}-x_{1} y_{2} y_{3} z_{4} \\
& -i\left(x_{3} x_{4} y_{2} z_{1}-x_{2} x_{3} y_{4} z_{1}-x_{3} x_{4} y_{1} z_{2}+x_{1} x_{3} y_{4} z_{2}+x_{2} x_{3} y_{1} z_{4}-x_{1} x_{3} y_{2} z_{4}\right), \\
& \Phi_{-+--}\left(\bar{r}_{1}, \bar{r}_{2}, \bar{r}_{3}, \bar{r}_{4}\right)=-\Phi_{+-++}^{*}\left(\bar{r}_{1}, \bar{r}_{2}, \bar{r}_{3}, \bar{r}_{4}\right) \\
& =-x_{4} y_{2} y_{3} z_{1}+x_{3} y_{2} y_{4} z_{1}+x_{4} y_{1} y_{2} z_{3}-x_{1} y_{2} y_{4} z_{3}-x_{3} y_{1} y_{2} z_{4}+x_{1} y_{2} y_{3} z_{4}
\end{aligned}
$$




$$
\begin{aligned}
& \quad+i\left(x_{2} x_{4} y_{3} z_{1}-x_{2} x_{3} y_{4} z_{1}-x_{2} x_{4} y_{1} z_{3}+x_{1} x_{2} y_{4} z_{3}+x_{2} x_{3} y_{1} z_{4}-x_{1} x_{2} y_{3} z_{4}\right), \\
& \Phi_{+---}\left(\bar{r}_{1}, \bar{r}_{2}, \bar{r}_{3}, \bar{r}_{4}\right)=-\Phi_{-+++}^{*}\left(\bar{r}_{1}, \bar{r}_{2}, \bar{r}_{3}, \bar{r}_{4}\right) \\
& =x_{4} y_{1} y_{3} z_{2}-x_{3} y_{1} y_{4} z_{2}-x_{4} y_{1} y_{2} z_{3}+x_{2} y_{1} y_{4} z_{3}+x_{3} y_{1} y_{2} z_{4}-x_{2} y_{1} y_{3} z_{4} \\
& \quad-i\left(x_{1} x_{4} y_{3} z_{2}-x_{1} x_{3} y_{4} z_{2}-x_{1} x_{4} y_{2} z_{3}+x_{1} x_{2} y_{4} z_{3}+x_{1} x_{3} y_{2} z_{4}-x_{1} x_{2} y_{3} z_{4}\right),
\end{aligned}
$$

where the superscripts $m_{i}= \pm 1 / 2$ are abbreviated to \pm . The above expressions are grouped into pairs. In the first three pairs, two spins are up and two down, and the spatial wave functions associated with a given spin state and the one obtained by flipping all spins are conjugate to each other. In the remaining four pairs, one spin is up and three are down, and the spatial wave function associated with a given spin state is minus the conjugate of the spatial wave function of the state with flipped spins.

With the notations

$$
\bar{r}_{i j}=\left(x_{i j}, y_{i j}, z_{i j}\right)=\bar{r}_{i} \times \bar{r}_{j}=\left(y_{i} z_{j}-y_{j} z_{i}, z_{i} x_{j}-z_{j} x_{i}, x_{i} y_{j}-y_{i} x_{j}\right),
$$

and

$$
\bar{r}_{i j, k l}=\left(x_{i j, k l}, y_{i j, k l}, z_{i j, k l}\right)=\bar{r}_{i j} \times \bar{r}_{k l},
$$

the two types of expressions can be reduced to

$$
\begin{array}{r}
\Phi_{--++}\left(\bar{r}_{1}, \bar{r}_{2}, \bar{r}_{3}, \bar{r}_{4}\right)=\Phi_{++--}^{*}\left(\bar{r}_{1}, \bar{r}_{2}, \bar{r}_{3}, \bar{r}_{4}\right)=\bar{r}_{12} \cdot \bar{r}_{34}+i z_{12,34}, \\
-\Phi_{-+-+}\left(\bar{r}_{1}, \bar{r}_{2}, \bar{r}_{3}, \bar{r}_{4}\right)=-\Phi_{+-+-}^{*}\left(\bar{r}_{1}, \bar{r}_{2}, \bar{r}_{3}, \bar{r}_{4}\right)=\bar{r}_{13} \cdot \bar{r}_{24}+i z_{13,24}, \\
\Phi_{-++-}\left(\bar{r}_{1}, \bar{r}_{2}, \bar{r}_{3}, \bar{r}_{4}\right)=\Phi_{+--+}^{*}\left(\bar{r}_{1}, \bar{r}_{2}, \bar{r}_{3}, \bar{r}_{4}\right)=\bar{r}_{14} \cdot \bar{r}_{23}+i z_{14,23}
\end{array}
$$

and

$$
\begin{aligned}
& \Phi_{---+}\left(\bar{r}_{1}, \bar{r}_{2}, \bar{r}_{3}, \bar{r}_{4}\right)=-\Phi_{+++-}^{*}\left(\bar{r}_{1}, \bar{r}_{2}, \bar{r}_{3}, \bar{r}_{4}\right)=\left(\bar{r}_{1} \cdot \bar{r}_{23}\right)\left(y_{4}-i x_{4}\right), \\
& -\Phi_{--+-}\left(\bar{r}_{1}, \bar{r}_{2}, \bar{r}_{3}, \bar{r}_{4}\right)=\Phi_{++-+}^{*}\left(\bar{r}_{1}, \bar{r}_{2}, \bar{r}_{3}, \bar{r}_{4}\right)=\left(\bar{r}_{1} \cdot \bar{r}_{24}\right)\left(y_{3}-i x_{3}\right), \\
& \Phi_{-+--}\left(\bar{r}_{1}, \bar{r}_{2}, \bar{r}_{3}, \bar{r}_{4}\right)=-\Phi_{+-++}^{*}\left(\bar{r}_{1}, \bar{r}_{2}, \bar{r}_{3}, \bar{r}_{4}\right)=\left(\bar{r}_{1} \cdot \bar{r}_{34}\right)\left(y_{2}-i x_{2}\right), \\
& -\Phi_{+---}\left(\bar{r}_{1}, \bar{r}_{2}, \bar{r}_{3}, \bar{r}_{4}\right)=\Phi_{-+++}^{*}\left(\bar{r}_{1}, \bar{r}_{2}, \bar{r}_{3}, \bar{r}_{4}\right)=\left(\bar{r}_{2} \cdot \bar{r}_{34}\right)\left(y_{1}-i x_{1}\right) .
\end{aligned}
$$

Therefore, the spatial probability density of the wave function (6), obtained by summing over the different possible spin configurations, is equal to

$$
\begin{aligned}
& \mathcal{P}_{0_{1}^{+}}\left(\bar{r}_{1}, \bar{r}_{2}, \bar{r}_{3}, \bar{r}_{4}\right) \\
& =\left(\frac{f}{r_{1} r_{2} r_{3} r_{4}}\right)^{2} \sum_{m_{i}} \Phi_{m_{1} m_{2} m_{3} m_{4}}\left(\bar{r}_{1}, \bar{r}_{2}, \bar{r}_{3}, \bar{r}_{4}\right) \Phi_{m_{1} m_{2} m_{3} m_{4}}^{*}\left(\bar{r}_{1}, \bar{r}_{2}, \bar{r}_{3}, \bar{r}_{4}\right) \\
& =\frac{3}{1024 \pi^{4}} \frac{1}{r_{1}^{2} r_{2}^{2} r_{3}^{2} r_{4}^{2}}
\end{aligned}
$$




$$
\begin{aligned}
& \times\left(\left(\bar{r}_{12} \cdot \bar{r}_{34}\right)^{2}+\left(\bar{r}_{13} \cdot \bar{r}_{24}\right)^{2}+\left(\bar{r}_{14} \cdot \bar{r}_{23}\right)^{2}+z_{12,34}^{2}+z_{13,24}^{2}+z_{14,23}^{2}\right. \\
& \quad+\left(x_{1}^{2}+y_{1}^{2}\right)\left(\bar{r}_{2} \cdot \bar{r}_{34}\right)^{2}+\left(x_{2}^{2}+y_{2}^{2}\right)\left(\bar{r}_{1} \cdot \bar{r}_{34}\right)^{2} \\
& \left.\quad+\left(x_{3}^{2}+y_{3}^{2}\right)\left(\bar{r}_{1} \cdot \bar{r}_{24}\right)^{2}+\left(x_{4}^{2}+y_{4}^{2}\right)\left(\bar{r}_{1} \cdot \bar{r}_{23}\right)^{2}\right) .
\end{aligned}
$$

This expression can be reduced further. From the identities

$$
\begin{aligned}
\bar{a} \times \bar{b} & =-\bar{b} \times \bar{a} \\
\bar{a} \cdot(\bar{b} \times \bar{c}) & =\bar{b} \cdot(\bar{c} \times \bar{a})=\bar{c} \cdot(\bar{a} \times \bar{b}) \\
(\bar{a} \times \bar{b}) \times(\bar{c} \times \bar{d}) & =-(\bar{a} \cdot(\bar{b} \times \bar{c})) \bar{d}+(\bar{a} \cdot(\bar{b} \times \bar{d})) \bar{c}
\end{aligned}
$$

it follows that

$$
\begin{aligned}
& x_{12,34}=-\left(\bar{r}_{1} \cdot \bar{r}_{23}\right) x_{4}+\left(\bar{r}_{1} \cdot \bar{r}_{24}\right) x_{3}=-x_{34,12}=\left(\bar{r}_{1} \cdot \bar{r}_{34}\right) x_{2}-\left(\bar{r}_{2} \cdot \bar{r}_{34}\right) x_{1}, \\
& x_{13,24}=+\left(\bar{r}_{1} \cdot \bar{r}_{23}\right) x_{4}+\left(\bar{r}_{1} \cdot \bar{r}_{34}\right) x_{2}=-x_{24,13}=\left(\bar{r}_{1} \cdot \bar{r}_{24}\right) x_{3}+\left(\bar{r}_{2} \cdot \bar{r}_{34}\right) x_{1}, \\
& x_{14,23}=+\left(\bar{r}_{1} \cdot \bar{r}_{24}\right) x_{3}-\left(\bar{r}_{1} \cdot \bar{r}_{34}\right) x_{2}=-x_{23,14}=\left(\bar{r}_{1} \cdot \bar{r}_{23}\right) x_{4}-\left(\bar{r}_{2} \cdot \bar{r}_{34}\right) x_{1} .
\end{aligned}
$$

Similar properties are valid for the $y$ and $z$ components. Furthermore, the following relation holds:

$$
\bar{r}_{1}\left(\bar{r}_{2} \cdot \bar{r}_{34}\right)-\bar{r}_{2}\left(\bar{r}_{1} \cdot \bar{r}_{34}\right)+\bar{r}_{3}\left(\bar{r}_{1} \cdot \bar{r}_{24}\right)-\bar{r}_{4}\left(\bar{r}_{1} \cdot \bar{r}_{23}\right)=0
$$

By combining the squares of the $x$ and $y$ components of this identity with the squares of the expressions (A.8) (and their equivalent expressions for the $y$ components), one obtains the relations

$$
\begin{aligned}
& x_{12,34}^{2}+x_{13,24}^{2}+x_{14,23}^{2} \\
& =x_{1}^{2}\left(\bar{r}_{2} \cdot \bar{r}_{34}\right)^{2}+x_{2}^{2}\left(\bar{r}_{1} \cdot \bar{r}_{34}\right)^{2}+x_{3}^{2}\left(\bar{r}_{1} \cdot \bar{r}_{24}\right)^{2}+x_{4}^{2}\left(\bar{r}_{1} \cdot \bar{r}_{23}\right)^{2}, \\
& y_{12,34}^{2}+y_{13,24}^{2}+y_{14,23}^{2} \\
& =y_{1}^{2}\left(\bar{r}_{2} \cdot \bar{r}_{34}\right)^{2}+y_{2}^{2}\left(\bar{r}_{1} \cdot \bar{r}_{34}\right)^{2}+y_{3}^{2}\left(\bar{r}_{1} \cdot \bar{r}_{24}\right)^{2}+y_{4}^{2}\left(\bar{r}_{1} \cdot \bar{r}_{23}\right)^{2} .
\end{aligned}
$$

With use of these identities in the expression (A.6), the latter can be further simplified to give

$$
\begin{aligned}
& \mathcal{P}_{0_{1}^{+}}\left(\bar{r}_{1}, \bar{r}_{2}, \bar{r}_{3}, \bar{r}_{4}\right) \\
& =\frac{3}{1024 \pi^{4}} \frac{1}{r_{1}^{2} r_{2}^{2} r_{3}^{2} r_{4}^{2}}\left(\left(\bar{r}_{12} \cdot \bar{r}_{34}\right)^{2}+\left(\bar{r}_{13} \cdot \bar{r}_{24}\right)^{2}+\left(\bar{r}_{14} \cdot \bar{r}_{23}\right)^{2}\right. \\
& \left.\quad+\left|\bar{r}_{12} \times \bar{r}_{34}\right|^{2}+\left|\bar{r}_{13} \times \bar{r}_{24}\right|^{2}+\left|\bar{r}_{14} \times \bar{r}_{23}\right|^{2}\right),
\end{aligned}
$$


which coincides with Eq. (11).

\section{B The angular probability density in relative angles $\theta_{i j}$. Derivation in spherical coordinates}

In this appendix we give the algorithm for the symbolic conversion of the angular probability density associated with the wave function (36) of four identical particles in a single- $\ell$ shell into an expression depending on the relative angles $\theta_{i j}$ only. Use is made of spherical coordinates. The starting point is the generic expression (41) which can be rewritten as

$$
\begin{aligned}
& \mathcal{P}_{\ell^{4}\left[L^{\prime} S^{\prime}, L^{\prime \prime} S^{\prime \prime}\right] L S}\left(\Omega_{1}, \Omega_{2}, \Omega_{3}, \Omega_{4}\right) \\
& =\sum_{\bar{r} \bar{s}} f_{\bar{r} \bar{s}}\left(\phi_{1}, \phi_{2}, \phi_{3}, \phi_{4}\right) \prod_{j=1}^{4}\left(\cos \theta_{j}\right)^{2 \ell-r_{j}-s_{j}}\left(\sin \theta_{j}\right)^{s_{j}},
\end{aligned}
$$

with

$$
f_{\bar{r} \bar{s}}\left(\phi_{1}, \phi_{2}, \phi_{3}, \phi_{4}\right)=\sum_{\bar{n}} a_{\bar{r} \bar{s} \bar{n}} \prod_{i=1}^{4}\left(\cos \phi_{i}\right)^{n_{i}}\left(\sin \phi_{i}\right)^{s_{i}-n_{i}},
$$

where the coefficients $a_{\bar{r} \bar{n} \bar{n}}$ can be obtained from the spatial probability density (38) after insertion of the explicit wave-function expression (36). The first step in the algorithm is to rewrite the functions $f_{\bar{r} \bar{s}}\left(\phi_{1}, \phi_{2}, \phi_{3}, \phi_{4}\right)$ in terms of $\cos \phi_{i j} \equiv \cos \left(\phi_{i}-\phi_{j}\right)$. In general, one can propose an expansion of the form

$$
\begin{aligned}
& f_{\bar{r} \bar{s}}\left(\phi_{1}, \phi_{2}, \phi_{3}, \phi_{4}\right) \equiv g_{\bar{r} \bar{s}}\left(\phi_{12}, \phi_{13}, \phi_{14}, \phi_{23}, \phi_{24}, \phi_{34}\right) \\
& =a_{\bar{r} \bar{s}}+\sum_{(i j)} a_{\bar{r} \bar{s}}^{i j} \cos \phi_{i j}+\sum_{(i j)(k l)} a_{\bar{r} \bar{s}}^{i j, k l} \cos \phi_{i j} \cos \phi_{k l} \\
& \quad+\sum_{(i j)(k l)(m n)} a_{\bar{r} \bar{s}}^{i j, k l, m n} \cos \phi_{i j} \cos \phi_{k l} \cos \phi_{m n} \\
& \quad+\sum_{(i j)(k l)(m n)(p q)} a_{\bar{r} \bar{s}}^{i j, k l, m n, p q} \cos \phi_{i j} \cos \phi_{k l} \cos \phi_{m n} \cos \phi_{p q}+\cdots
\end{aligned}
$$

where the summations are over pairs $(i j),(k l), \ldots$ with $i \neq j, k \neq l, \ldots$ The coefficients $a_{\bar{r} \bar{s}}, a_{\bar{r} \bar{s}}^{i j}, \ldots$ obviously depend on the coefficients $a_{\bar{r} \bar{s} \bar{n}}$ in the definition of $f_{\bar{r} \bar{s}}\left(\phi_{1}, \phi_{2}, \phi_{3}, \phi_{4}\right)$ but the structure of the expansion is also determined by the set $\bar{s}$ from general arguments. The number of times $\phi_{i}$ occurs in Eq. (B.1) is $s_{i}$ and it may not occur the same number of times in Eq. (B.3). The expansion (B.3) should be such, however, that in each term $\phi_{i}$ occurs $s_{i}-2 k$ times (with $k=0,1, \ldots, \frac{1}{2} s_{i}$ ) and the exponents can then be matched by multiplying this term with $\left(\sin ^{2} \phi_{i}+\cos ^{2} \phi_{i}\right)^{k}$. For example, if $\bar{s}=\{2222\}$, the expansion acquires the form ${ }^{4}$

$\overline{4}$ The subscript $\bar{r}$ is dropped since it does not enter into this discussion. 


$$
\begin{aligned}
& g_{\{2222\}}\left(\phi_{12}, \phi_{13}, \phi_{14}, \phi_{23}, \phi_{24}, \phi_{34}\right) \\
& =a_{\{2222\}}+\sum_{(i j)} a_{\{2222\}}^{i j, i j} \cos ^{2} \phi_{i j}+\sum_{(i j)(k l)(m n)} a_{\{2222\}}^{i j, k l, m n} \cos \phi_{i j} \cos \phi_{k l} \cos \phi_{m n} \\
& \quad+\sum_{(i j)(k l)(m n)(p q)} a_{\{2222\}}^{i j, k l, m n, p q} \cos \phi_{i j} \cos \phi_{k l} \cos \phi_{m n} \cos \phi_{p q},
\end{aligned}
$$

where the first sum has six terms [(12), (13), (14), (23), (24) and (34)], the second sum has three terms $[(12)(13)(23),(12)(14)(24)$ and $(23)(24)(34)]$ and the third sum has six terms $[(12)(12)(34)(34),(12)(13)(24)(34),(12)(14)(23)(34)$, $(13)(13)(24)(24),(13)(14)(23)(24)$ and $(14)(14)(23)(23)]$. From general arguments certain coefficients (like $a_{\{2222\}}^{i j}$ ) must vanish. To give another example, if $\bar{s}=\{2211\}$, the expansion becomes

$$
\begin{aligned}
& g_{\{2211\}}\left(\phi_{12}, \phi_{13}, \phi_{14}, \phi_{23}, \phi_{24}, \phi_{34}\right) \\
& =a_{\{2211\}}^{34} \cos \phi_{34}+\sum_{(i j)(k l)} a_{\{2211\}}^{i j, k l} \cos \phi_{i j} \cos \phi_{k l} \\
& +\sum_{(i j)(k l)(m n)} a_{\{2211\}}^{i j, k l, m n} \cos \phi_{i j} \cos \phi_{k l} \cos \phi_{m n}
\end{aligned}
$$

where the first sum has two terms $[(13)(14)$ and $(23)(24)]$ and the second sum has three terms $[(12)(12)(34),(12)(13)(24)$ and $(12)(14)(23)]$. For each of the functions $f_{\bar{r} \bar{s}}\left(\phi_{1}, \phi_{2}, \phi_{3}, \phi_{4}\right)$ in the sum (B.1) - they are 41 in number in the $p$ shell-an equivalent function $g_{\bar{r} \bar{s}}\left(\phi_{12}, \phi_{13}, \phi_{14}, \phi_{23}, \phi_{24}, \phi_{34}\right)$ can be determined by writing $\cos \phi_{i j}$ in Eq. (B.3) in terms of the cosine and the sine of the individual angles $\phi_{i}$ and $\phi_{j}$, and equating the result to Eq. (B.1). Given a set of coefficients $a_{\bar{r} \bar{s} \bar{n}}$, these equations can be solved for the unknowns $a_{\bar{r} \bar{s}}, a_{\bar{r} \bar{s}}^{i j}, \ldots$ and this determines the function $g_{\bar{r} \bar{s}}\left(\phi_{12}, \phi_{13}, \phi_{14}, \phi_{23}, \phi_{24}, \phi_{34}\right)$. In fact, in general there are several possible forms $g_{\bar{r} \bar{s}}^{\alpha}\left(\phi_{12}, \phi_{13}, \phi_{14}, \phi_{23}, \phi_{24}, \phi_{34}\right), \alpha=1,2, \ldots$ for the function and it is imperative that all possibilities are found for the subsequent step (the conversion to the angles $\theta_{i j}$ ) to work. It is always feasible to find all possibilities since the relation between the coefficients $a_{\bar{r} \bar{s} \bar{n}}$ and $a_{\bar{r} \bar{s}}$, $a_{\bar{r} \bar{s}}^{i j}, \ldots$ is linear. As a result of the first step, the substitution

$$
f_{\bar{r} \bar{s}}\left(\phi_{1}, \phi_{2}, \phi_{3}, \phi_{4}\right) \mapsto \sum_{\alpha} u_{\bar{r} \bar{s}}^{\alpha} g_{\bar{r} \bar{s}}^{\alpha}\left(\phi_{12}, \phi_{13}, \phi_{14}, \phi_{23}, \phi_{24}, \phi_{34}\right)
$$

is made in the angular probability density (B.1), where $u_{\bar{r} \bar{s}}^{\alpha}$ are unknowns, to be determined later, that satisfy $\sum_{\alpha} u_{\bar{r} \bar{s}}^{\alpha}=1$.

In the second step of the algorithm, the dependence on the azimuthal angles $\phi_{i}$ is eliminated with use of the identity

$$
\cos \phi_{i j}=\frac{\cos \theta_{i j}-\cos \theta_{i} \cos \theta_{j}}{\sin \theta_{i} \sin \theta_{j}} .
$$

The resulting expression for the angular probability density depends on the 
relative angles $\theta_{i j}$, which is what we want to achieve, but still also on the polar angles $\theta_{i}$, which should be avoided. After this substitution, the following generic expression is obtained for the angular probability density:

$$
\sum_{\bar{q} \bar{p}} b_{\bar{q} \bar{p}}\left(\prod_{(i j) \in \bar{q}-\bar{p}} \cos \theta_{i j}\right)\left(\prod_{k=1}^{4}\left(\cos \theta_{k}\right)^{q_{k}-p_{k}}\right),
$$

where $\bar{q}$ and $\bar{p}$ are short-hand notations for sets of four integers $\left\{q_{1} q_{2} q_{3} q_{4}\right\}$ and $\left\{p_{1} p_{2} p_{3} p_{4}\right\}$, respectively. For the $p$ shell the possible sets for $\bar{q}$ are: $\{2222\}$ (1), $\{2220\}(4),\{2200\}(6),\{2000\}$ (4) and $\{0000\}$ (1), where the number in brackets after each set indicates the number of possible permutations, so that the sum over $\bar{q}$ contains in fact 16 terms. Furthermore, the values of $p_{i}$ are obtained by taking $1,2, \ldots$ pairs out of the set $\bar{q}$. For example, if $\bar{q}=\{2222\}$, the possible sets for $\bar{p}$ are: $\{2222\}(1 \times 1),\{2211\}(1 \times 6),\{2200\}(1 \times 6)$, $\{2110\}(1 \times 12),\{1111\}(3 \times 1),\{2000\}(1 \times 4),\{1100\}(2 \times 6)$ and $\{0000\}$ $(6 \times 1)$. The brackets after each set contain products of two numbers; the first gives the number of ways the pairs can be selected and the second indicates the number of possible permutations. So, for example, to obtain $\bar{p}=\{2211\}$ from $\bar{q}=2222$ one necessarily must select the pair (34) and there are six possible permutations $(\{2211\},\{2121\},\{1221\},\{2112\},\{1212\}$ and $\{1122\})$. As another example, there is only one permutation of $\bar{p}=\{1111\}$ but it can be obtained from $\bar{q}=\{2222\}$ by taking out the pairs $(12)(34),(13)(24)$ or $(14)(23)$. The expression for the angular probability density is now obtained by requiring that all coefficients $b_{\bar{q} \bar{p}}$ with $\bar{p} \neq \bar{q}$ vanish. This leads to a set of

equations in the unknowns $u_{\bar{r} \bar{s}}^{\alpha}$ introduced earlier, which generally is woefully overcomplete. For arbitrary coefficients $a_{\bar{r} \bar{s} \bar{n}}$ in the original expression (B.1), it allows no solution but for a genuine angular probability density it should. In fact, it was found in the $p$ shell that the equations $b_{\bar{q} \bar{p}}=0$ always leave one unknown $u_{\bar{r} \bar{s}}^{\alpha}$ unspecified which can be chosen at will.

This algorithm can be implemented in a symbolic computer language such as Mathematica, enabling the automatic conversion of a probability density depending on the polar and azimuthal angles $\Omega_{i}$ to one which depends on the relative angles $\theta_{i j}$.

\section{References}

[1] P. Mei and P. Van Isacker, previous paper.

[2] M.V. Zhukov, B.V. Danilin, D.V. Fedorov, J.M. Bang, I.J. Thompson and J.S. Vaagen, Phys. Reports 231 (1993) 151.

[3] M.V. Zhukov, A.A. Korsheninnikov and M.H. Smedberg, Phys. Rev. C 50 (1994) R1. 
[4] K. Hagino, N. Takahashi and H. Sagawa, Phys. Rev. C 77 (2008) 054317.

[5] H. Hancock, Theory of Maxima and Minima, Ginn and Company, ??, 1917.

[6] N. Keeley et al., Phys. Lett. B 646 (2007) 222.

[7] I. Talmi, Simple Models of Complex Nuclei. The Shell Model and Interacting Boson Model, Harwood, Chur, 1993.

[8] R. Gilmore, Catastrophe Theory for Scientists and Engineers, Wiley, New York, 1981.

[9] S. Cohen and D. Kurath, Nucl. Phys. A 101 (1967) 1. 